\title{
Opérations de Hausdorff itérées et réunions croissantes de compacts
}

\author{
par \\ Sylvain Kahane (Paris)
}

\begin{abstract}
In this paper, motivated by questions in Harmonic Analysis, we study the operation of (countable) increasing union, and show it is not idempotent: $\omega_{1}$ iterations are needed in general to obtain the closure of a class under this operation.

Increasing union is a particular Hausdorff operation, and we present the combinatorial tools which allow to study the power of various Hausdorff operations, and of their iterates. Besides countable increasing union, we study in detail a related Hausdorff operation, which preserves compactness.
\end{abstract}

Introduction. L'origine de ce travail se situe dans la tradition de l'étude des classes d'ensembles minces apparaissant naturellement en Analyse Harmonique et de leur stabilité par les opérations de réunions finies ou dénombrables. Une partie de ce travail est exposé dans ma Thèse de Doctorat dirigée par Alain Louveau [6].

Le problème initial est l'étude de la stabilité par réunion dénombrable croissante de diverses classes d'ensembles minces; la question de la stabilité par réunion croissante de la classe des ensembles de convergence absolue a été posée par J. Arbault en 1952 [2]. Ce problème est résolu dans [7] gràce aux outils développés ici.

Pour construire le stabilisé par l'opération de réunion croissante (dénombrable) d'une classe $\mathcal{C}$ donnée, considérons les réunions de suites croissantes (pour l'inclusion) d'éléments de $\mathcal{C}$; elles constituent une nouvelle classe, notée $\mathcal{C}^{\uparrow}$, qui n'est pas, en général, stable par réunion croissante. Mais en itérant $\omega_{1}$ fois l'opération $\uparrow$, nous obtenons la plus petite classe stable par réunion croissante contenant $\mathcal{C}$. B. Aniszczyk, J. Burzyk et A. Kamiński ont montré l'existence d'une classe pour laquelle il est nécessaire d'itérer $\omega_{1}$ fois l'opération $\uparrow$ avant d'obtenir le stabilisé par réunion croissante [1]. Nous démontrerons à nouveau ce résultat par deux méthodes très différentes. La première (Partie 3) utilise un autre théorème de hiérarchie, le théorème de BorelLebesgue sur les classes de Baire, et est proche de celle de B. Aniszczyk, 
J. Burzyk et A. Kamiński. La deuxième (Partie 4) est purement combinatoire et permet d'obtenir un résultat plus fort.

L'opération de réunion croissante est plus compliquée que les opérations de réunions finie ou dénombrable d'un point de vue combinatoire (c'est la présente étude) et d'un point de vue descriptif. En effet, dans [3], H. Becker, $\mathrm{S}$. Kahane et A. Louveau étudient la complexité descriptive de la classe $\mathcal{C}^{\uparrow}$ pour une classe borélienne $\mathcal{C}$ de compacts et montrent qu'il n'existe pas en général de procédé simple (borélien) permettant d'obtenir à partir d'un élément compact de $\mathcal{C}^{\uparrow}$ une suite croissante d'éléments de $\mathcal{C}$ dont il est la réunion.

Notre objectif principal étant de montrer que des classes existantes ne sont pas stables par réunion croissante, nous donnerons un procédé pour construire, à partir d'une classe $\mathcal{C}$ donnée, des éléments dans les itérés de $\mathcal{C}$ par l'opération $\uparrow$. Nous introduirons, en particulier, une opération, notée $\mathcal{P}$, qui permet de construire des boréliens de petite classe de Baire dans les itérés de $\mathcal{C}$. L'opération $\mathcal{P}$ à la propriété forte de transformer les suites de compacts en compacts. L'opération $\mathcal{P}$ étant liée à l'opération $\uparrow$, elle permet de construire des compacts dans les itérés d'une classe de compacts par l'opération de réunion croissante compacte et de montrer l'existence d'une classe pour laquelle il est nécessaire d'itérer $\omega_{1}$ fois l'opération de réunion croissante compacte avant d'obtenir le stabilisé. Ce procédé de construction de compacts suivant un rang transfini diffère complètement des procédés classiques tels que celui de G. Cantor pour construire des compacts nécessitant une dérivation transfinie pour extraire la partie parfaite ou celui de A. S. Kechris, A. Louveau et W. H. Woodin [8] pour construire des compacts de rang coanalytique quelconque dans les $\sigma$-idéaux de compacts.

Pour une classe héréditaire, l'opération de réunion croissante est équivalente à l'opération de limite inférieure ensembliste. Nous serons amenés à considérer des limites inférieures itérées que nous interpréterons comme des opérations de Hausdorff dont la base est un filtre de Fréchet itéré. Nous verrons que les comparaisons d'opérations de Hausdorff suivant diverses bases monotones se ramènent à des problèmes combinatoires sur les bases. Nous introduirons une classe canonique $\mathcal{I}$ qui ne possède aucune propriété de stabilité par les opérations de Hausdorff. La classe $\mathcal{I}$ contient une suite d'éléments parfaitement indépendants sur laquelle peut être comparée la puissance respective de deux opérations de Hausdorff. Une classe ayant cette propriété est appelée antistable. Dans [7], on démontre l'existence de nombreuses classes antistables naturelles d'ensembles minces, comme la classe des ensembles de convergence absolue ou celle des compacts de Dirichlet fort. Les méthodes permettant de passer de la classe $\mathcal{I}$ à des classes d'ensembles minces demande des outils spécifiques à l'Analyse Harmonique qu'il était souhaitable de developper distinctement. 
Nous étudierons également l'opération de réunion asymptotique, i.e., l'opération de Hausdorff de base le filtre $\mathcal{A} s$ des parties d'entiers de densité 1, qui est liée à la limite inférieure. Il est courant en Analyse Harmonique de considérer, pour une classe donnée $\mathcal{C}$, la classe $\mathcal{W C}$ des parties dont les mesures sont portées à $\varepsilon$ près par des éléments de $\mathcal{C}$ (par exemple, la classe $\mathcal{W D}$ des ensembles de Dirichlet faibles). Or la classe $\mathcal{W C}$ est stable par l'opération $\mathcal{A} s$. Nous avons ainsi un procédé pour construire des éléments de $\mathcal{W C}$. Nous verrons qu'il existe une classe pour laquelle il est nécessaire d'itérer $\omega_{1}$ fois l'opération $\mathcal{A} s$ avant d'obtenir le stabilisé par réunion asymptotique (Partie 5).

Dans la Partie 1, nous rappelons quelques propriétés simples des opérations de Hausdorff qui, bien que très connues, ne se trouvent pas facilement dans la littérature. Les parties suivantes sont consacrées aux lemmes combinatoires de comparaisons entre itérées d'opérations de Hausdorff, et aux applications aux opérations de réunion croissante, de réunion croissante compacte et de réunion asymptotique.

\section{Résultats généraux sur les opérations de Hausdorff de base monotone}

a) Définitions. On désignera par $\omega$ (resp. $\left.\omega_{1}\right)$ le premier ordinal infini (resp. non dénombrable). L'ensemble des entiers naturels sera également noté $\omega$.

On identifiera $\mathcal{P}(E)$, l'ensemble des parties de $E$, avec $2^{E}$ par la bijection $A \mapsto 1_{A}$; en particulier, la partie vide de $E$ sera notée $\emptyset$ ou 0 .

Définition 1.1. On dira qu'une partie $\mathcal{F}$ de $2^{\omega} \backslash\{0\}$ est une base monotone sur $\omega$, si $\mathcal{F}$ est non vide et monotone (ou cohéréditaire) (i.e., $[A \in \mathcal{F}$ et $A \subset B] \Rightarrow B \in \mathcal{F})$.

DÉfinition 1.2. Soient $\mathcal{F}$ une base monotone sur $\omega$ et $\left(X_{n}\right)_{n \in \omega}$ une suite de parties d'un ensemble $E$. L'opération de Hausdorff de base $\mathcal{F}$ est l'opération qui à toute suite $\left(X_{n}\right)_{n \in \omega}$ associe l'ensemble, noté $H_{\mathcal{F}}\left(X_{n}\right)$, des $x \in E$ tels que $\left\{n \in \omega ; x \in X_{n}\right\} \in \mathcal{F}$. (Si $\mathcal{F}$ est le filtre de Fréchet, on obtient la limite inférieure ensembliste.)

Si $\mathcal{C}$ est une classe héréditaire (i.e., $[B \in \mathcal{C}$ et $A \subset B] \Rightarrow A \in \mathcal{C}$ ) de parties de $E$, on note $\mathcal{C}^{\mathcal{F}}$ la classe des images de suites d'éléments de $\mathcal{C}$ par l'opération de Hausdorff de base $\mathcal{F}$, c'est-à-dire

$$
\mathcal{C}^{\mathcal{F}}=\left\{H_{\mathcal{F}}\left(X_{n}\right) ;\left(X_{n}\right)_{n \in \omega} \subseteq \mathcal{C}\right\} .
$$

L'opération $\mathcal{F}$ désignera l'opération sur les classes $\mathcal{C} \mapsto \mathcal{C}^{\mathcal{F}}$.

Si $\mathcal{C}^{\mathcal{F}}=\mathcal{C}$, on dira que $\mathcal{C}$ est une $\mathcal{F}$-classe (de parties de $E$ ). 
Nous allons donner des exemples de bases monotones avec les opérations correspondantes. Dans la suite, $\mathcal{C}$ désignera une classe héréditaire de parties d'un ensemble $E$.

Exemples. 1) Posons $\sigma=2^{\omega} \backslash\{0\}$.

$$
\begin{aligned}
\mathcal{C}^{\sigma} & =\left\{X \in \mathcal{P}(E) ; \exists\left(X_{n}\right)_{n \in \omega} \subseteq \mathcal{C}, X \subseteq \bigcup_{n \in \omega} X_{n}\right\} \\
& =\text { le } \sigma \text {-idéal engendré par } \mathcal{C} .
\end{aligned}
$$

2) Nous noterons $\mathcal{F} r$ le filtre de Fréchet sur $\omega$, c'est-à-dire l'ensemble des parties cofinies de $\omega$. Pour toute suite $\left(X_{n}\right)_{n \in \omega}$, on a $H_{\mathcal{F r} r}\left(X_{n}\right)=$ $\bigcup_{m \in \omega} \bigcap_{n \geq m} X_{n}$. Comme la classe $\mathcal{C}$ est héréditaire, la classe $\mathcal{C}^{\mathcal{F r}}$ est égale à la classe

$$
\mathcal{C}^{\uparrow}=\left\{X \in \mathcal{P}(E) ; \exists\left(X_{n}\right)_{n \in \omega} \text { croissante } \subseteq \mathcal{C}, X \subseteq \bigcup_{n \in \omega} X_{n}\right\}
$$

Attention, $\mathcal{C}^{\uparrow}$ n'est pas, en général, la $\uparrow$-classe (ou $\mathcal{F} r$-classe) engendrée $\operatorname{par} \mathcal{C}$.

3) Posons $\mathcal{U}^{(n)}=\left\{A \in 2^{\omega} ; A \cap[0, n-1] \neq \emptyset\right\}(n \in \omega)$.

$$
\begin{aligned}
& \mathcal{C}^{\mathcal{U}^{(n)}}=\left\{X \in \mathcal{P}(E) ; \exists\left(X_{i}\right)_{0 \leq i \leq n-1} \subseteq \mathcal{C}, X \subseteq \bigcup_{i=0}^{n-1} X_{i}\right\}, \\
& \mathcal{C}^{\mathcal{U}^{(1)}}=\mathcal{C} .
\end{aligned}
$$

Notons $\mathcal{U}=\mathcal{U}^{(2)}$; les $\mathcal{U}$-classes sont les idéaux (i.e., les classes stables par réunions finies).

4) Nous noterons $\mathcal{P}$ l'ensemble des parties coponctuelles de $\omega$, c'est-àdire des parties de complémentaire de cardinal $\leq 1$. On a $\mathcal{P} \subset \mathcal{F} r$. Pour toute suite $\left(X_{n}\right)_{n \in \omega}, H_{\mathcal{P}}\left(X_{n}\right)=\bigcup_{m \in \omega} \bigcap_{n \neq m} X_{n}$.

Proposition 1.3. Si $\left(X_{n}\right)_{n \in \omega}$ est une suite de compacts d'un compact métrisable $E$, alors $X=H_{\mathcal{P}}\left(X_{n}\right)$ est compact. Plus généralement, si $\left(X_{n}\right)_{n \in \omega}$ est une suite de boréliens d'un espace polonais $E$ appartenant à la même classe de Baire multiplicative $\Pi_{\alpha}^{0}$ (voir Partie 3 pour une définition plus précise), alors $X=H_{\mathcal{P}}\left(X_{n}\right) \in \Pi_{\alpha}^{0}$.

Démonstration. Pour tout $x \in E$, on a

$$
x \in X \Leftrightarrow \forall m \in \omega\left(x \in X_{m} \text { ou }\left[\forall n \neq m, x \in X_{n}\right]\right) .
$$

L'ensemble $X$ est donc compact (resp. $\Pi_{\alpha}^{0}$ ), car la classe des compacts (resp. la classe $\Pi_{\alpha}^{0}$ ) est stable par intersections dénombrables. 
5) Nous noterons $\mathcal{A} s$ l'ensemble des parties de densité 1 de $\omega$, c'est-à-dire des parties $A$ de $\omega$ telles que

$$
\mathrm{d}(A)=\lim _{n \rightarrow \infty} \frac{\operatorname{card}(A \cap[0, n-1])}{n}=1 .
$$

On a $\mathcal{F} r \subset \mathcal{A} s$ et $\mathcal{A} s$ est un $\mathcal{K}_{\sigma \delta}$ de $2^{\omega}$. Pour toute suite $\left(X_{n}\right)_{n \in \omega}$,

$$
H_{\mathcal{A} s}\left(X_{n}\right)=\left\{x \in E ; \lim _{n \rightarrow \infty} \frac{1}{n} \sum_{k=1}^{n} 1_{X_{n}}(x)=1\right\} \text {. }
$$

Donnons un exemple de $\mathcal{A} s$-classe.

Proposition 1.4. Si $\mathcal{C}$ est une classe héréditaire de compacts d'un compact métrisable $E$, alors la classe $\mathcal{W C}$ des parties universellement mesurables $X$ de $E$ telles que

$$
\forall \mu \in \mathcal{M}^{+}(E), \forall \varepsilon>0, \exists K \in \mathcal{C}, \quad \mu(X \backslash K)<\varepsilon
$$

(où $\mathcal{M}^{+}(E)$ est l'ensemble des mesures boréliennes positives sur $E$ ) est une As-classe.

Ce résultat est une conséquence simple du théorème de convergence dominée de Lebesgue [7]. Remarquons que $\mathcal{W C}$ n'est pas un idéal en général (par exemple $\mathcal{W D}$ la classe des ensembles de Dirichlet faibles).

b) Comparaison. Considérons les "îlots" $I_{n}=\left\{A \in 2^{\omega} ; n \in A\right\}(n \in \omega)$; ce sont des ouvert-fermés de $2^{\omega}$ pour la topologie produit. Posons

$$
\mathcal{I}=\bigcup_{n \in \omega} \mathcal{P}\left(I_{n}\right)
$$

Nous allons voir que $\mathcal{I}$ est l'exemple canonique de classe héréditaire dans la comparaison des opérations de Hausdorff de base monotone.

Proposition 1.5. Soient $\mathcal{F}$ et $\mathcal{G}$ deux bases monotones sur $\omega$. Les propriétés suivantes sont équivalentes.

(a) $\exists \varphi: \omega \rightarrow \omega, \varphi^{-1} \mathcal{F} \subseteq \mathcal{G}$.

(b) $\forall E$ ensemble, $\forall \mathcal{C}$ héréditaire $\subseteq \mathcal{P}(E), \mathcal{C}^{\mathcal{F}} \subseteq \mathcal{C}^{\mathcal{G}}$.

(c) $\mathcal{F} \in \mathcal{I}^{\mathcal{G}}$.

Si le couple $(\mathcal{F}, \mathcal{G})$ vérifie les propriétés précédentes, on note $\mathcal{F} \preceq \mathcal{G}$.

Démonstration. Montrons que (a) entraîne (b). Soient $E$ un ensemble, $\mathcal{C} \subseteq \mathcal{P}(E)$ et $\left(X_{n}\right)_{n \in \omega} \subseteq \mathcal{C}$. Soit $x \in E$; si $x \in H_{\mathcal{F}}\left(X_{n}\right)$, alors $\left\{n \in \omega ; x \in X_{n}\right\} \in \mathcal{F}$. D'après (a), $\varphi^{-1}\left\{n \in \omega ; x \in X_{n}\right\}=\{k \in \omega ; x \in$ $\left.X_{\varphi(k)}\right\} \in \mathcal{G}$, donc $x \in H_{\mathcal{G}}\left(X_{\varphi(k)}\right)$. On a bien $\mathcal{C}^{\mathcal{F}} \subseteq \mathcal{C}^{\mathcal{G}}$.

Remarquons que $\mathcal{F}=H_{\mathcal{F}}\left(I_{n}\right)$, donc $\mathcal{F} \in \mathcal{I}^{\mathcal{F}}$, et (c) est un cas particulier de (b).

Supposons (c); il existe une suite $\left(X_{k}\right)_{k \in \omega} \subseteq \mathcal{I}$ telle que $\mathcal{F}=H_{\mathcal{G}}\left(X_{k}\right)$. D'après la définition de $\mathcal{I}$, il existe une application $\varphi: \omega \rightarrow \omega$ telle que $X_{k} \subseteq$ 
$I_{\varphi(k)}(k \in \omega)$. Mais $\mathcal{F} \subseteq H_{\mathcal{G}}\left(I_{\varphi(k)}\right)$, car $\mathcal{G}$ est monotone. Si $A \in H_{\mathcal{G}}\left(I_{\varphi(k)}\right)$, alors $\varphi^{-1}(A)=\left\{k \in \omega ; A \in I_{\varphi(k)}\right\} \in \mathcal{G}$, donc $\varphi^{-1} \mathcal{F} \subseteq \mathcal{G}$. Ceci ferme la boucle.

Nous allons faire plusieurs remarques sur la relation $\preceq$.

Remarques. 1) La relation $\preceq$ est un préordre (i.e., une relation reflexive et transitive) sur la famille des bases monotones sur $\omega$.

On notera $\prec$ le préordre strict défini par $\mathcal{F} \prec \mathcal{G} \Leftrightarrow(\mathcal{F} \preceq \mathcal{G}$ et $\mathcal{G} \npreceq \mathcal{F})$, $\equiv$ la relation d'équivalence définie par $\mathcal{F} \equiv \mathcal{G} \Leftrightarrow(\mathcal{F} \preceq \mathcal{G}$ et $\mathcal{G} \preceq \mathcal{F})$, et $\perp$ la relation "être étranger à" définie par $\mathcal{F} \perp \mathcal{G} \Leftrightarrow(\mathcal{F} \npreceq \mathcal{G}$ et $\mathcal{G} \npreceq \mathcal{F})$.

2) $\mathcal{U}^{(1)}$ est le plus petit élément du préordre $\preceq$ modulo $\equiv$ et $\mathcal{U}^{(1)} \equiv\{\omega\}$.

3) $\mathcal{P}$ est le successeur immédiat et unique, modulo $\equiv$, de $\mathcal{U}^{(1)}$ pour le préordre $\preceq[7]$.

4) $\sigma$ est le plus grand élément du préordre $\preceq$ modulo $\equiv$.

5) On a $\mathcal{P} \prec \mathcal{F} r \prec \mathcal{A} s, \mathcal{F} r \perp \mathcal{U}$ et $\mathcal{A} s \perp \mathcal{U}$.

6) On a $\mathcal{P} \prec \mathcal{U}$, et, plus généralement, si $\mathcal{G}$ est une base monotone sur $\omega$, compacte pour la topologie produit sur $2^{\omega}$, alors $\mathcal{G} \preceq \mathcal{U}^{(n)}$, pour un certain $n \in \omega$.

En effet, sinon pour tout $n \in \omega$, il existe un $A_{n} \in \mathcal{G}$ tel que $A_{n} \cap$ $[0, n-1]=\emptyset$. Mais $A_{n} \rightarrow 0$ et $0 \notin \mathcal{G}$.

7) Il n'existe pas de base monotone $\mathcal{F}$ telle que pour toute classe héréditaire $\mathcal{C}$, la classe $\mathcal{C}^{\mathcal{F}}$ soit l'idéal engendré par $\mathcal{C}$.

En effet, sinon $\mathcal{F} \in \mathcal{I}^{\mathcal{F}}=\bigcup_{n \in \omega} \mathcal{I}^{\mathcal{U}^{(n)}}$, et $\mathcal{F} \preceq \mathcal{U}^{(n)}$, pour un $n \in \omega$, ce qui est une contradiction puisque les $\mathcal{U}^{(n)}$ sont strictement croissants pour $\prec$.

c) Dualité

DÉfinition 1.6. A toute partie $\mathcal{F}$ de $2^{\omega}$, on associe son dual $\widehat{\mathcal{F}}$ défini par

$$
\widehat{\mathcal{F}}=\left\{A \in 2^{\omega} ; A^{c} \in \mathcal{F}^{c}\right\} .
$$

Re marque. Soit $\mathcal{F}$ une base monotone sur $\omega,\left(X_{n}\right)_{n \in \omega}$ une suite de parties de $E$ et $x \in E$; alors

$$
x \notin H_{\mathcal{F}}\left(X_{n}\right) \Leftrightarrow\left\{n \in \omega ; x \notin X_{n}\right\} \in \widehat{\mathcal{F}} .
$$

ExEmples. Calculons les duaux des exemples usuels : $\widehat{\sigma}=\{\omega\}, \widehat{\mathcal{F} r}$ est l'ensemble des parties infinies de $\omega, \widehat{\mathcal{A} s}=\left\{A \in 2^{\omega} ; \operatorname{ds}(A)=\right.$ $\limsup _{n \rightarrow \infty} \operatorname{card}\left(A \cap[0, n[) / / n>0\}, \widehat{\mathcal{U}^{(1)}}=\mathcal{U}^{(1)}, \widehat{\mathcal{U}^{(n)}}=\left\{A \in 2^{\omega} ;[0, n[\right.\right.$ $\subseteq A\}$ et $\widehat{\mathcal{P}}=\left\{A \in 2^{\omega} ; \operatorname{card}(A) \geq 2\right\}$.

Remarquons que la dualité n'est pas compatible avec $\preceq$, puisque $\widehat{\mathcal{A s}} \equiv$ $\widehat{\mathcal{F} r} \equiv\{\widehat{\omega}\}$.

Rappelons qu'un filtre (resp. une grille) est une base monotone vérifiant $[(A \in \mathcal{F}$ et $B \in \mathcal{F}) \Rightarrow A \cap B \in \mathcal{F}]($ resp. $[A \cup B \in \mathcal{F} \Rightarrow(A \in \mathcal{F}$ ou $B \in \mathcal{F})])$. 
Proposition 1.7. (1) Pour toute partie $\mathcal{F}$ de $2^{\omega}$, on a :

(a) $\widehat{\hat{\mathcal{F}}}=\mathcal{F}$.

(b) $\mathcal{F}$ est monotone si et seulement si $\forall A \in \mathcal{F}, \forall B \in \widehat{\mathcal{F}}, A \cap B \neq \emptyset$. En particulier, si $\mathcal{F}$ est une base monotone sur $\omega$, alors $\widehat{\mathcal{F}}=\left\{B \in 2^{\omega} ; \forall A \in\right.$ $\mathcal{F}, A \cap B \neq \emptyset\}$ et $\widehat{\mathcal{F}}$ est une base monotone sur $\omega$.

(c) $\mathcal{F}$ est un filtre si et seulement si $\widehat{\mathcal{F}}$ est une grille. Dans ce cas, $\mathcal{F} \subseteq \widehat{\mathcal{F}}$.

(2) Soient $\mathcal{F}$ et $\mathcal{G}$ deux bases monotones sur $\omega$ et $\varphi: \omega \rightarrow \omega$. Alors, $\varphi^{-1} \mathcal{F} \subseteq \mathcal{G}$ si et seulement si $\varphi \widehat{\mathcal{G}} \subseteq \widehat{\mathcal{F}}$. En particulier,

$$
\mathcal{F} \npreceq \mathcal{G} \Leftrightarrow \forall \varphi: \omega \rightarrow \omega, \exists A \in \widehat{\mathcal{G}}, \varphi(A)^{c} \in \mathcal{F} .
$$

Les propositions de (1) sont classiques [4] et la démonstration de (2) ne présente pas de difficulté (à noter que le fait que $\mathcal{F}$ et $\mathcal{G}$ soient monotones est essentiel).

d) Composition. Nous allons voir que la composée de deux opérations de Hausdorff est encore une opération de Hausdorff. Fixons une bijection \langle\rangle$: \omega^{2} \rightarrow \omega$. Posons, pour tout $A \in 2^{\omega}$ et $n \in \omega$,

$$
A_{n}=\{k \in \omega ;\langle n, k\rangle \in A\} .
$$

DÉfinition 1.8. Soient $\mathcal{F}$ et $\mathcal{G}$ deux bases monotones sur $\omega$. On pose

$$
\mathcal{F} \otimes \mathcal{G}=\left\{A \in 2^{\omega} ;\left\{n \in \omega ; A_{n} \in \mathcal{G}\right\} \in \mathcal{F}\right\} .
$$

Remarquons que $\mathcal{F} \otimes \mathcal{G}$ est définie à une permutation sur $\omega$ près, ce qui n’influera pas sur les propriétés élémentaires qui suivent.

Proposition 1.9. Soient $\mathcal{F}$ et $\mathcal{G}$ deux bases monotones sur $\omega$.

(a) $\mathcal{F} \otimes \mathcal{G}$ est une base monotone sur $\omega$.

(b) Soient $E$ un ensemble et $\left(X_{n}\right)_{n \in \omega} \subseteq \mathcal{P}(E)$; alors

$$
H_{\mathcal{F} \otimes \mathcal{G}}\left(X_{n}\right)=H_{n \rightarrow \mathcal{F}}\left[H_{k \rightarrow \mathcal{G}}\left(X_{\langle n, k\rangle}\right)\right] .
$$

En particulier, si $\mathcal{C} \subseteq \mathcal{P}(E)$, alors $\mathcal{C}^{\mathcal{F} \otimes \mathcal{G}}=\left(\mathcal{C}^{\mathcal{G}}\right)^{\mathcal{F}}$.

(c) La loi $\otimes$ est associative.

(d) La loi $\otimes$ est compatible avec la relation $\preceq$.

(e) $\widehat{\mathcal{F} \otimes} \mathcal{G}=\widehat{\mathcal{F}} \otimes \widehat{\mathcal{G}}$.

Proposition 1.10. $\mathcal{U} \otimes \mathcal{F} r \prec \mathcal{F} r \otimes \mathcal{U}$, i.e., il existe une classe $\mathcal{C}$ telle que $\left(\mathcal{C}^{\uparrow}\right)^{\mathcal{U}}$ soit inclus strictement dans $\left(\mathcal{C}^{\mathcal{U}}\right)^{\uparrow}$. En particulier, la loi $\otimes$ est non commutative.

R e marque. Dans la pratique on visualisera $\mathcal{F} \otimes \mathcal{G}$ comme un sousensemble de $\omega^{2}$. 


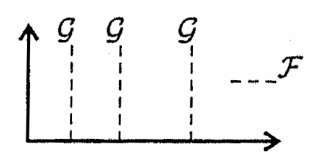

Démonstration. Pour tout $A \in 2^{\omega}$, on a

$$
\begin{aligned}
& A \in \widehat{\mathcal{U}} \otimes \widehat{\mathcal{F}} r\left\{n \in \omega ; A_{n} \in \widehat{\mathcal{F} r}\right\} \in \widehat{\mathcal{U}} \Leftrightarrow A_{0} \text { et } A_{1} \text { infinis }, \\
& A \in \widehat{\mathcal{F} r} \otimes \widehat{\mathcal{U}} \Leftrightarrow\left\{n \in \omega ; A_{n} \in \widehat{\mathcal{U}}\right\} \in \widehat{\mathcal{F} r} \\
& \\
& \Leftrightarrow A_{n} \supseteq\{0,1\} \text { pour une infinité de } n \in \omega .
\end{aligned}
$$

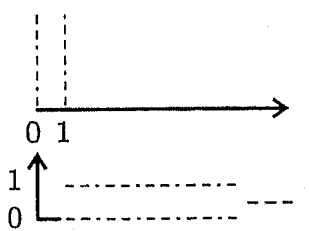

Considérons $\varphi: \omega^{2} \rightarrow \omega^{2},(n, k) \mapsto(k, n)$. Comme $\varphi(\widehat{\mathcal{F} r} \otimes \widehat{\mathcal{U}}) \subseteq \widehat{\mathcal{U}} \otimes \widehat{\mathcal{F} r}$, on a $\mathcal{U} \otimes \mathcal{F} r \preceq \mathcal{F} r \otimes \mathcal{U}$.

Inversement, montrons que pour tout $\varphi: \omega^{2} \rightarrow \omega^{2}$, il existe un $A \in$ $\widehat{\mathcal{U}} \otimes \widehat{\mathcal{F} r}$ tel que $\varphi(A) \notin \widehat{\mathcal{F} r} \otimes \widehat{\mathcal{U}}$.

Considérons l'application $\varphi_{\lceil 1}$ qui à $u \in \omega^{2}$ associe la première coordonnée de $\varphi(u)$. Si $\varphi_{\lceil 1}(\{0\} \times \omega)$ ou $\varphi_{\lceil 1}(\{1\} \times \omega)$ est fini, il suffit de prendre $A=\{0,1\} \times \omega$. Sinon, on peut choisir $A_{0}$ et $A_{1}$ infinis tels que $\varphi_{\lceil 1}\left(\{0\} \times A_{0}\right) \cap \varphi_{\lceil 1}\left(\{1\} \times A_{1}\right)=\emptyset$ et que les applications $f_{i}: A_{i} \rightarrow \omega$, $k \mapsto \varphi_{\lceil 1}(i, k)(i=0,1)$ soient injectives; alors $A=\bigcup_{i=0,1}\{i\} \times A_{i}$ convient.

En conclusion, $\mathcal{F} r \otimes \mathcal{U} \npreceq \mathcal{U} \otimes \mathcal{F} r$.

Re marque. On a même $\mathcal{P} \otimes \mathcal{U} \npreceq \mathcal{U} \otimes \mathcal{F} r$.

2. Itérations d'opérations de Hausdorff. En général, le stabilisé d'une classe de parties par une opération de Hausdorff n'est pas obtenu du premier coup et il est nécessaire d'itérer l'opération de Hausdorff.

DÉfinition 2.1. Soient $\mathcal{F}$ une base monotone sur $\omega$ et $\mathcal{C}$ une classe héréditaire de parties d'un ensemble $E$. Définissons, par récurrence sur l'ordinal $\alpha$, la classe $\mathcal{C}^{\alpha \mathcal{F}}$ :

$$
\mathcal{C}^{0 \mathcal{F}}=\mathcal{C}, \quad \mathcal{C}^{<\alpha \mathcal{F}}=\bigcup_{\beta<\alpha} \mathcal{C}^{\beta \mathcal{F}}, \quad \mathcal{C}^{\alpha \mathcal{F}}=\left(\mathcal{C}^{<\alpha \mathcal{F}}\right)^{\mathcal{F}}, \quad \mathcal{C}^{\infty \mathcal{F}}=\mathcal{C}^{<\omega_{1} \mathcal{F}}
$$

Le rang de $\mathcal{C}$ pour l'opération $\mathcal{F}$ est $\operatorname{rg}_{\mathcal{F}}(\mathcal{C})=\inf \left\{\alpha \leq \omega_{1} ; \mathcal{C}^{\alpha \mathcal{F}}=\mathcal{C}^{\infty \mathcal{F}}\right\}$ et la hauteur de $\mathcal{F}$ est $\operatorname{ht}(\mathcal{F})=\sup \left\{\operatorname{rg}_{\mathcal{F}}(\mathcal{C}) ; \mathcal{C}\right\}$.

Remarques. 1) $\mathcal{C}^{\infty \mathcal{F}}$ est une $\mathcal{F}$-classe; c'est le stabilisé de $\mathcal{C}$ par l'opération $\mathcal{F}$. 
2) $\operatorname{ht}(\{\omega\})=0, \operatorname{ht}(\sigma)=1, \operatorname{ht}(\mathcal{U})=\omega$.

3) Pour tout $n \in \omega, \mathcal{C}^{n \mathcal{F}}=\mathcal{C} \underbrace{\mathcal{F} \otimes \ldots \otimes \mathcal{F}}_{n \text { fois }}$.

4) En général, pour $\alpha$ infini, $\mathcal{C}^{\alpha \mathcal{F}}$ ne s'obtient pas par l'opération d'une base monotone sur la classe : par exemple, $\mathcal{C}^{\omega \mathcal{U}}$, qui est l'idéal engendré par $\mathcal{C}$, ne peut être obtenu par une telle opération (Remarque 1.b.7).

Nous allons voir que néanmoins les bases monotones sur $\omega$ s'introduisent naturellement dans l'étude de $\mathcal{C}^{\alpha \mathcal{F}}$.

On appelle suite fondamentale associée à l'ordinal dénombrable $\alpha \geq 1$ toute suite $\left([\alpha]_{n}\right)_{n \in \omega}$ vérifiant : si $\alpha=\beta+1,[\alpha]_{n}=\beta(n \in \omega)$; si $\alpha$ est un ordinal limite, $[\alpha]_{n} \nearrow \alpha$.

DÉfinition 2.2. A tout ordinal $\alpha$, on associe un arbre $T_{\alpha}$ de $\omega^{<\omega}$ étiqueté par des ordinaux $\leq \alpha$ et défini par $T_{0}=\{\emptyset\}$ et

$$
\forall \alpha \geq 1, \forall n \in \omega, \forall u \in \omega^{<\omega}, \quad\left(n^{\wedge} u \in T_{\alpha} \Leftrightarrow u \in T_{[\alpha]_{n}}\right) .
$$

L'étiquette en $u \in T_{\alpha}$ est notée $[\alpha]_{u}$ et est définie par

$$
[\alpha]_{\emptyset}=\alpha, \quad[\alpha]_{u^{\wedge} n}=\left[[\alpha]_{u}\right]_{n} \quad\left(u \in \omega^{<\omega}, n \in \omega\right) .
$$

On a dans la définition de $T_{\alpha}$ associé implicitement une suite fondamentale à tout ordinal (ce sont les étiquettes de ses fils dans l'arbre); un même ordinal pouvant apparaître plusieurs fois, on s'autorise à chaque apparition un choix différent de la suite fondamentale.

Dès que $\alpha$ est infini, le choix de $T_{\alpha}$ (ou de son squelette) n'est pas unique.

Remarque. $T_{\alpha}$ est un arbre bien fondé (i.e., sans branches infinies) de hauteur $\alpha$.

DÉfinition 2.3. On note $\widetilde{T}_{\alpha}$ l'ensemble des nœuds extérieurs de $T_{\alpha}$. En particulier, $\widetilde{T}_{1}=\omega$ et $\widetilde{T}_{\alpha+1}=\omega \times \widetilde{T}_{\alpha}$.

Soit \langle\rangle une bijection de $\omega^{2}$ dans $\omega$. Nous construisons par récurrence sur $\alpha \geq 2$ une bijection \langle\rangle$_{\alpha}$ de $\widetilde{T}_{\alpha}$ dans $\omega$ par :

$$
\langle\rangle_{2}=\langle\rangle \text { et }\left\langle n^{\wedge} u\right\rangle_{\alpha}=\left\langle\left(n,\langle u\rangle_{[\alpha]_{n}}\right)\right\rangle \quad\left(\alpha \geq 3, n \in \omega, u \in \omega^{<\omega}\right) .
$$

DÉfinition 2.4. Soient $\alpha$ un ordinal dénombrable $\geq 1, A$ une partie de $\widetilde{T}_{\alpha}$ et $\mathcal{F}$ une base monotone sur $\omega$. Le jeu $G(\mathcal{F}, \alpha, A)$ est un jeu fini entièrement déterminé à deux joueurs I et II. Le joueur I commence et joue des parties $B_{i} \in \mathcal{F}$. Le joueur II répond par un entier $n_{i} \in B_{i}$. La partie s'arrète quand la suite $\left(n_{0}, \ldots, n_{k}\right)$ des coups joués par II appartient à $\widetilde{T}_{\alpha}$ ( $T_{\alpha}$ est un arbre bien fondé, donc toute partie s'arrète). Enfin, I gagne la 
partie, si $\left(n_{0}, \ldots, n_{k}\right) \in A$.

$$
\begin{array}{cc}
\mathrm{I} & \mathrm{II} \\
B_{0} \in \mathcal{F} & \\
& n_{0} \in B_{0} \\
B_{1} \in \mathcal{F} & \\
\vdots & n_{1} \in B_{1} \\
B_{k} \in \mathcal{F} & \vdots \\
& n_{k} \in B_{k}
\end{array}
$$

On appelle $\mathcal{F}_{\alpha}$ l'ensemble des parties $A$ de $\widetilde{T}_{\alpha}$ (resp. de $\omega$ ) telles que I ait une stratégie gagnante dans le jeu $G(\mathcal{F}, \alpha, A)\left(\operatorname{resp} . G\left(\mathcal{F}, \alpha,\langle\rangle_{\alpha}^{-1} A\right)\right)$.

Remarque. La définition de $\mathcal{F}_{\alpha}$ dépend du choix de $T_{\alpha}$ : il n'y a pas a priori unicité de $\mathcal{F}_{\alpha}$ modulo la relation d'équivalence $\equiv$ associée à $\preceq$ (Proposition 2.11). Quand il y a lieu de distinguer les différents $\mathcal{F}_{\alpha}$, nous les noterons $\mathcal{F}_{T_{\alpha}}$. Nous verrons dans les Propositions 2.8 et 2.10 que pour $\mathcal{F} r$ et $\mathcal{A} s$ les itérés ne dépendent pas du choix de $T_{\alpha}$.

Proposition 2.5. (a) $\mathcal{F}_{\alpha}$ est une base monotone sur $\omega$.

(b) Si $\mathcal{F}$ est un filtre (borélien), alors $\mathcal{F}_{\alpha}$ est un filtre (borélien).

(c) $\mathcal{F}_{1}=\mathcal{F}$ et $\mathcal{F}_{\alpha+1}=\mathcal{F} \otimes \mathcal{F}_{\alpha}$.

(d) Si $\mathcal{F}$ est borélienne, $\widehat{\mathcal{F}}_{\alpha}=\widehat{\mathcal{F}}_{\alpha}=\left\{A \subseteq \widetilde{T}_{\alpha}\right.$; II a une stratégie gagnante dans le jeu $\left.G\left(\mathcal{F}, \alpha, A^{c}\right)\right\}$.

Démonstration. (a), (b) et (c) se déduisent directement des différentes définitions.

Clairement $\widehat{\mathcal{F}}_{\alpha}=\left\{A ; A^{c} \notin \mathcal{F}_{\alpha}\right\}=\{A$; II a une stratégie gagnante dans le jeu $\left.G\left(\mathcal{F}, \alpha, A^{c}\right)\right\}$, puisque le jeu $G\left(\mathcal{F}, \alpha, A^{c}\right)$ est borélien, donc déterminé (i.e., l'un des deux joueurs a une stratégie gagnante) (théorème de D. A. Martin). Soit $A \in \widehat{\mathcal{F}_{\alpha}}$ et $s$ une stratégie gagnante pour II dans le jeu $G\left(\mathcal{F}, \alpha, A^{c}\right)$ ne dépendant que des coups précédents de II et du dernier coup de I (on remarquera qu'une telle stratégie gagnante existe bien); on pose

$$
s^{\prime}\left(n_{0}, \ldots, n_{i-1}\right)=B_{i}=\left\{s\left(n_{0}, \ldots, n_{i-1}, C_{i}\right)=n_{i} ; C_{i} \in \mathcal{F}\right\} .
$$

Alors $A \in \widehat{\mathcal{F}}_{\alpha}$, car $s^{\prime}$ est une stratégie gagnante pour I dans $G(\widehat{\mathcal{F}}, \alpha, A)$ ne dépendant que des précédents coups de II; en effet, $B_{i} \in \widehat{\mathcal{F}}$, car $B_{i} \cap C \neq \emptyset$, pour tout $C \in \mathcal{F}$, et si $n_{i} \in B_{i},\left(n_{0}, \ldots, n_{i}\right)$ est le début d'un élément de $A$. Inversement, si $A \in \widehat{\mathcal{F}}_{\alpha}$ et $s^{\prime}$ est une stratégie gagnante pour I dans le jeu $G(\widehat{\mathcal{F}}, \alpha, A)$ ne dépendant que des précédents coups de II, on vérifiera que $s\left(n_{0}, \ldots, n_{i-1}, C_{i}\right)=n_{i}$ où $n_{i} \in B_{i} \cap C_{i}$ avec $B_{i}=s^{\prime}\left(n_{0}, \ldots, n_{i-1}\right)$ est une stratégie gagnante pour II dans le jeu $G\left(\mathcal{F}, \alpha, A^{c}\right)$.

Proposition 2.6. Pour toute classe $\mathcal{C}, \mathcal{C}^{\alpha \mathcal{F}}$ est la réunion, sur les différents choix de $T_{\alpha}$, des $\mathcal{C}^{\mathcal{F}_{T_{\alpha}}}$. En particulier, $\operatorname{ht}(\mathcal{F})=\operatorname{rg}_{\mathcal{F}}(\mathcal{I})$. 
Démonstration. Montrons le résultat par récurrence sur $\alpha$. Si $X \in$ $\mathcal{C}^{\alpha \mathcal{F}}$, alors $X \subseteq H_{\mathcal{F}}\left(X_{n}\right)$ avec $X_{n} \in \mathcal{C}^{\alpha_{n} \mathcal{F}}\left(\alpha_{n}<\alpha\right)$. Quitte à grandir les $\alpha_{n}$, on peut supposer que $\left(\alpha_{n}\right)_{n \in \omega}$ est une suite fondamentale associée à $\alpha$; la construction d'un bon $T_{\alpha}$ est immédiate.

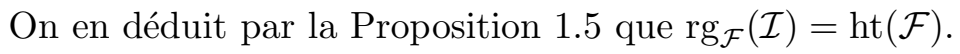

Proposition 2.7. Soit $\mathcal{F}$ une base monotone sur $\omega$. Pour tout ordinal $\alpha \leq \operatorname{ht}(\mathcal{F})$, il existe une classe héréditaire $\mathcal{C} \subset \mathcal{P}\left(2^{\omega}\right)$ telle que $\operatorname{rg}_{\mathcal{F}}(\mathcal{C})=\alpha$.

Démonstration. Comme $\operatorname{rg}_{\mathcal{F}}(\mathcal{I})=\operatorname{ht}(\mathcal{F})$, pour tout $\alpha<\operatorname{ht}(\mathcal{F})$, alors il existe $A \in \mathcal{I}^{\alpha \mathcal{F}} \backslash \mathcal{I}^{<\alpha \mathcal{F}}$. D'après la proposition précédente, il existe une suite $\left(B_{n}\right)_{n \in \omega}$ d'éléments de $\mathcal{I}$ telle que $A=H_{\mathcal{F}_{\alpha}}\left(B_{n}\right)$. Posons $A_{n}=$ $B_{n} \cap A(n \in \omega)$. Alors, $A=H_{\mathcal{F}_{\alpha}}\left(A_{n}\right)$; en effet, si $x \in A$, alors $\{n ; x \in$ $\left.A_{n}\right\}=\left\{n ; x \in B_{n}\right\}$.

Posons $\mathcal{C}=\bigcup_{n \in \omega} \mathcal{P}\left(A_{n}\right)$. Alors $\mathcal{C}$ est contenue dans $\mathcal{I} \cap \mathcal{P}(A)$ et $A \in$ $\mathcal{C}^{\alpha \mathcal{F}}$, donc $\mathcal{C}^{\alpha \mathcal{F}}=\mathcal{P}(A)=\mathcal{C}^{\infty \mathcal{F}}$, car $\mathcal{P}(A)$ est une $\mathcal{F}$-classe. Inversement $\mathcal{C} \subset \mathcal{I}$, donc $A \notin \mathcal{C}^{<\alpha \mathcal{F}}$. On a bien $\operatorname{rg}_{\mathcal{F}}(\mathcal{C})=\alpha$.

Revenons au cas du filtre de Fréchet.

On sait que $\mathcal{C}^{\mathcal{F} r}=\mathcal{C}^{\uparrow}=\left\{X \in \mathcal{P}(E) ; \exists\left(X_{n}\right)_{n \in \omega}\right.$ croissante $\subseteq \mathcal{C}, X \subseteq$ $\left.\bigcup_{n \in \omega} X_{n}\right\}$. Nous noterons $\mathcal{C}^{\alpha \uparrow}=\mathcal{C}^{\alpha \mathcal{F} r}$.

Proposition 2.8. Quel que soit le choix de $T_{\alpha}, \mathcal{C}^{\alpha \uparrow}=\mathcal{C}^{\mathcal{F} r_{T_{\alpha}}}$. En particulier, il existe un unique filtre de Fréchet $\alpha$-itéré $\mathcal{F} r_{\alpha}$ modulo $\equiv$.

Démonstration. Considérons un choix de $T_{\alpha}$, correspondant à une suite fondamentale $\left([\alpha]_{n}\right)_{n \in \omega}$. Soit $\left(X_{n}\right)_{n \in \omega}$ une suite de parties telle que $X_{n} \in \mathcal{C}^{\alpha_{n} \mathcal{F}}\left(\alpha_{n}<\alpha\right)$. Il existe une application $\varphi: \omega \rightarrow \omega$ croissante surjective telle que $\alpha_{\varphi(n)} \leq[\alpha]_{n}(n \in \omega)$. Alors, on a $H_{\mathcal{F} r}\left(X_{n}\right) \subseteq H_{\mathcal{F} r}\left(X_{\varphi(n)}\right)$. On en déduit, par induction sur $\alpha$, le résultat.

Le résultat peut être également obtenu pour le filtre $\mathcal{A} s$.

Lemme 2.9. Soit $\mathcal{F}$ une base monotone sur $\omega$ et $\alpha<\omega_{1}$. Si $\left(\alpha_{n}\right)_{n \in \omega}$ et $\left(\alpha_{n}^{\prime}\right)_{n \in \omega}$ sont deux choix d'une suite fondamentale pour $\alpha$ correspondant $\grave{a}$ deux arbres $T_{\alpha}$ et $T_{\alpha}^{\prime}$, alors la condition

$$
\exists f: \omega \rightarrow \omega,(\forall A \in \widehat{\mathcal{F}}, f(A) \in \widehat{\mathcal{F}}) \text { et }\left(\forall n \in \omega, \alpha_{f(n)}^{\prime} \leq \alpha_{n}\right)
$$

entraîne $\mathcal{F}_{T_{\alpha}^{\prime}} \preceq \mathcal{F}_{T_{\alpha}}$.

Démonstration. Soit $f: \omega \rightarrow \omega$ telle que pour tout $A \in \widehat{\mathcal{F}}$, on ait $f(A) \in \widehat{\mathcal{F}}$ et pour tout $n \in \omega, \alpha_{f(n)}^{\prime} \leq \alpha_{n}$. Alors, pour tout $n \in \omega$, $\mathcal{F}_{\alpha_{f(n)}^{\prime}} \preceq \mathcal{F}_{\alpha_{n}}$, donc il existe une application $\varphi_{n}: T_{\alpha_{n}} \rightarrow T_{\alpha_{f(n)}^{\prime}}$ telle que pour tout $A_{n} \in \widehat{\mathcal{F}}_{\alpha_{n}}$, on ait $\varphi_{n}\left(A_{n}\right) \in \widehat{\mathcal{F}}_{\alpha_{f(n)}^{\prime}}$. 
Considérons l'application $\varphi: T_{\alpha} \rightarrow T_{\alpha}^{\prime}$ de la forme $\varphi\left(n^{\wedge} u\right)=$ $f(n)^{\wedge} \varphi_{n}(u)$; on a immédiatement que pour tout $A \in \widehat{\mathcal{F}}_{T_{\alpha}}, \varphi(A) \in \widehat{\mathcal{F}}_{T_{\alpha}^{\prime}}$, donc $\mathcal{F}_{T_{\alpha}^{\prime}} \preceq \mathcal{F}_{T_{\alpha}}$.

Proposition 2.10. Quel que soit le choix de $T_{\alpha}, \mathcal{C}^{\alpha \mathcal{A} s}=\mathcal{C}^{\mathcal{A} s_{T_{\alpha}}}$. En particulier, il existe un unique filtre $\alpha$-itéré $\mathcal{A} s_{\alpha}$ modulo $\equiv$.

Dé m o n s trat i on. D'après le lemme précédent, il suffit de montrer que pour tout $\alpha<\omega_{1}$ et tout choix $\left(\alpha_{n}\right)_{n \in \omega}$ et $\left(\alpha_{n}^{\prime}\right)_{n \in \omega}$ de suite fondamentale pour $\alpha$ correspondant à deux arbres $T_{\alpha}$ et $T_{\alpha}^{\prime}$, il existe une application $f: \omega \rightarrow \omega$ telle que $\forall A \in \widehat{\mathcal{A} s}, f(A) \in \widehat{\mathcal{A} s}$ et $\forall n \in \omega, \alpha_{f(n)}^{\prime} \leq \alpha_{n}$.

On pourra considérer $g(p)=\inf \left\{n \in \omega ; \alpha_{p}^{\prime} \leq \alpha_{n}\right\}$, puis $g^{\prime}(p)=$ $\inf \left\{2 g^{\prime}(p-1), g(p)\right\}$ et poser $f(n)=\inf \left\{k \in \omega ; k+g^{\prime}(p) \equiv n(\bmod p)\right\}$, si $g^{\prime}(p) \leq n<g^{\prime}(p+1)$. On a $f(n)<p$ avec $g(p) \leq n$, donc $\alpha_{f(n)}^{\prime} \leq \alpha_{n}$ pour tout $n \in \omega$. De plus, les fibres de $f$ sont suffisament réparties pour que $\forall A \in \widehat{\mathcal{A}} s, f(A) \in \widehat{\mathcal{A}} s$; la vérification est laissée au lecteur.

Proposition 2.11. Il n'y a pas unicité des $\omega$-itérés de $\mathcal{P}$.

Démonstration. Si $\alpha<\omega_{1}$ et si $\left(\alpha_{n}\right)_{n \in \omega}$ et $\left(\alpha_{n}^{\prime}\right)_{n \in \omega}$ sont deux choix d'une suite fondamentale pour $\alpha$ correspondant à deux arbres $T_{\alpha}$ et $T_{\alpha}^{\prime}$, alors pour $\mathcal{P}$, la condition $(*)$ devient

$$
\exists f: \omega \rightarrow \omega \text { injective telle que }\left(\forall n \in \omega, \alpha_{f(n)}^{\prime} \leq \alpha_{n}\right) .
$$

Cette condition ne peut pas être vérifiée pour $\alpha=\omega$ et $\alpha_{0}=0, \alpha_{1}=3$, $\alpha_{0}^{\prime}=1, \alpha_{1}^{\prime}=2$ et $\alpha_{n}=\alpha_{n}^{\prime}=n+2$ pour $n \geq 2$. Dans le cas particulier où $\mathcal{F}=\mathcal{P}$ et $\alpha=\omega$, on peut démontrer la réciproque du Lemme 1.9, et on en déduit que $\mathcal{P}_{T_{\omega}^{\prime}} \perp \mathcal{P}_{T_{\omega}}$.

Question. Existe-t-il un filtre borélien pour lequel il n'y aurait pas unicité du filtre $\omega$-itéré ?

3. Théorèmes de hiérarchie et classes de Baire. Les exemples de problèmes où une opération doit être itérée $\omega_{1}$ fois sont nombreux en Analyse (dérivation des compacts par Cantor, totalisation des fonctions dérivées par Denjoy ...). Nous allons démontrer de nouveaux théorèmes de hiérarchie sur les itérées d'une opération de Hausdorff dont la base est un filtre borélien libre, en nous ramenant au théorème de Borel-Lebesgue sur la hiérarchie des classes de Baire.

Dans les paragraphes suivants, nous exposerons des résultats plus fins, liant les itérées de deux opérations de Hausdorff distinctes, dont les démonstrations combinatoires ne peuvent être a priori déduites de théorèmes de hiérarchie déjà existants.

Donnons le résultat principal de ce paragraphe qui est une conséquence du Corollaire 3.5 et de la Proposition 2.7. 
ThÉORÈme 3.1. Pour tout ordinal $\alpha \leq \omega_{1}$, il existe une classe de parties d'un ensemble dont le stabilisé par réunion croissante est obtenu par exactement $\alpha$ itérations de l'opération $\uparrow$.

B. Aniszczyk, J. Burzyk et A. Kamiński ont également montré l'existence d'une classe pour laquelle il est nécessaire d'itérer $\omega_{1}$ fois l'opération $\uparrow$ pour obtenir le stabilisé par réunion croissante [1]. Le schéma de leur démonstration est proposé à la fin de ce paragraphe.

Soit $E$ un espace polonais (i.e., métrisable séparable complet). Pour tout ordinal dénombrable $\alpha \geq 1$, on note $\Sigma_{\alpha}^{0}$ (resp. $\Pi_{\alpha}^{0}$, resp. $\Delta_{\alpha}^{0}$ ) la classe de Baire additive (resp. multiplicative, resp. ambigüe) des boréliens d'ordre $\alpha$ :

$$
\begin{aligned}
\Sigma_{1}^{0} & \equiv \text { ouvert, } \Sigma_{2}^{0} \equiv \mathcal{F}_{\sigma}, \Sigma_{3}^{0} \equiv \mathcal{G}_{\delta \sigma}, \ldots \\
\Pi_{1}^{0} & \equiv \text { fermé, } \Pi_{2}^{0} \equiv \mathcal{G}_{\delta}, \Pi_{3}^{0} \equiv \mathcal{F}_{\sigma \delta}, \ldots \\
\Delta_{\alpha}^{0} & =\Sigma_{\alpha}^{0} \cap \Pi_{\alpha}^{0} .
\end{aligned}
$$

ThÉORÈme (E. Borel-H. Lebesgue [9], [10]). Si E est un espace polonais non dénombrable, pour tout ordinal $\alpha \geq 1, \Sigma_{\alpha}^{0} \neq \Pi_{\alpha}^{0}$, i.e., pour tout couple d'ordinaux $(\alpha, \beta)$, avec $1 \leq \alpha<\beta<\omega_{1}, \Delta_{\beta}^{0} \backslash \Delta_{\alpha}^{0} \neq \emptyset$.

DÉfinition 3.2. Soient $\mathcal{F}$ un filtre et $\left(X_{n}\right)_{n \in \omega}$ une suite de parties d'un ensemble $E$. On note traditionnellement

$$
H_{\mathcal{F}}\left(X_{n}\right)=\liminf _{\mathcal{F}}\left(X_{n}\right) \quad \text { et } \quad H_{\widehat{\mathcal{F}}}\left(X_{n}\right)=\limsup _{\mathcal{F}}\left(X_{n}\right) .
$$

Cette notation se justifie car, comme $\mathcal{F} \subseteq \widehat{\mathcal{F}}$, on a

$$
\liminf _{\mathcal{F}}\left(X_{n}\right) \subseteq \limsup _{\mathcal{F}}\left(X_{n}\right) .
$$

On dit que $X \in \mathcal{P}(E)$ est limite suivant $\mathcal{F}$ de la suite $\left(X_{n}\right)_{n \in \omega}$ et on note $X=\lim _{\mathcal{F}}\left(X_{n}\right)$, si $\liminf \operatorname{Fin}_{\mathcal{F}}\left(X_{n}\right)=\lim \sup _{\mathcal{F}}\left(X_{n}\right)=X$.

Si $\mathcal{C}$ est une classe de parties de $E$, on note $\lim _{\mathcal{F}}(\mathcal{C})$ la classe des limites suivant $\mathcal{F}$ de suites d'éléments de $\mathcal{C}$.

Exemples. Pour le filtre de Fréchet, on retrouve la convergence au sens ensembliste :

$$
\begin{aligned}
X=\lim _{\mathcal{F} r}\left(X_{n}\right) & \Leftrightarrow X=\bigcup_{m \in \omega} \bigcap_{n \geq m} X_{n}=\bigcap_{m \in \omega} \bigcup_{n \geq m} X_{n} \\
& \Leftrightarrow\left(1_{X_{n}}\right)_{n \in \omega} \text { converge ponctuellement vers } 1_{X}
\end{aligned}
$$

et pour $\mathcal{A} s$, la convergence en moyenne de Cesàro :

$$
X=\lim _{\mathcal{A} s}\left(X_{n}\right) \Leftrightarrow 1_{X}=\lim _{n} \frac{1}{n} \sum_{k=0}^{n} 1_{X_{k}} .
$$


Lemme 3.3. Soient $E$ un espace polonais de dimension 0 et $\alpha$ un ordinal dénombrable $\geq 1$. On a

$$
\Delta_{\alpha+1}^{0}=\lim _{\mathcal{F} r_{\alpha}}\left(\Delta_{1}^{0}\right) .
$$

Dé m o n s t r a t i o n. L'inclusion de gauche à droite se déduit directement d'un théorème de $\mathrm{K}$. Kuratowski (Théorème 30.4 de [9]). La réciproque s'obtient en remarquant que, pour toute suite $\left(X_{n}\right)_{n \in \omega}, \liminf _{\mathcal{F}_{r}}\left(X_{n}\right) \subseteq$ $\lim \sup _{\mathcal{F} r}\left(X_{n}\right)$, donc $\lim _{\mathcal{F} r_{\alpha}}\left(X_{n}\right)$ est obtenue par une opération $\alpha$-itérée, où l'on peut alterner limites inférieures et limites supérieures de manière à obtenir la classe de Baire la plus petite : en effet, si $\left(X_{n}\right)_{n \in \omega} \subset \Pi_{\alpha}^{0}$, alors $\liminf \operatorname{if}_{\mathcal{F}}\left(X_{n}\right) \in \Sigma_{\alpha+1}^{0}$, et si $\left(X_{n}\right)_{n \in \omega} \subset \Sigma_{\alpha}^{0}$, alors $\lim \sup _{\mathcal{F} r}\left(X_{n}\right) \in \Pi_{\alpha+1}^{0}$.

Lemme 3.4. Soient $\mathcal{F}$ et $\mathcal{G}$ deux bases monotones sur $\omega$ et $\mathcal{C}$ une classe de parties (non nécessairement héréditaire). Alors

$$
\mathcal{F} \preceq \mathcal{G} \Rightarrow \lim _{\mathcal{F}}(\mathcal{C}) \subseteq \lim _{\mathcal{G}}(\mathcal{C}) .
$$

Démonstration. Si $\mathcal{F} \preceq \mathcal{G}$, alors il existe une application $\varphi$ de $\omega$ dans $\omega$ telle que

$$
\forall B \in 2^{\omega}, \quad\left(B \in \mathcal{F} \Rightarrow \varphi^{-1}(B) \in \mathcal{G}\right) .
$$

Mais, on a également (Proposition 1.7(2)) :

$$
\forall A \in 2^{\omega}, \quad(A \in \widehat{\mathcal{G}} \Rightarrow \varphi(A) \in \widehat{\mathcal{F}}) .
$$

On en déduit que si $\left(X_{n}\right)_{n \in \omega}$ est une suite d'éléments de $\mathcal{C}$, alors

$$
\liminf _{\mathcal{F}}\left(X_{n}\right) \subseteq \liminf _{\mathcal{G}}\left(X_{\varphi(k)}\right) \subseteq \limsup _{\mathcal{G}}\left(X_{\varphi(k)}\right) \subseteq \limsup _{\mathcal{F}}\left(X_{n}\right),
$$

$\operatorname{donc} \lim _{\mathcal{F}}\left(X_{n}\right)=\lim _{\mathcal{G}}\left(X_{\varphi(k)}\right)$ et $\lim _{\mathcal{F}}(\mathcal{C}) \subseteq \lim _{\mathcal{G}}(\mathcal{C})$.

Corollaire 3.5. La suite $\left(\mathcal{F} r_{\alpha}\right)_{\alpha<\omega_{1}}$ est strictement croissante pour $\prec$.

Démonstration. Sinon, il existe deux ordinaux $\alpha$ et $\beta$, avec $1 \leq \alpha$ $<\beta<\omega_{1}$, tels que $\mathcal{F} r_{\beta} \preceq \mathcal{F} r_{\alpha}$. D'après le Lemme 3.4, on a $\lim _{\mathcal{F} r_{\beta}}\left(\Delta_{1}^{0}\right)$ $\subseteq \lim _{\mathcal{F} r_{\alpha}}\left(\Delta_{1}^{0}\right)$, donc $\Delta_{\beta}^{0} \subseteq \Delta_{\alpha}^{0}$, ce qui contredit le théorème de BorelLebesgue.

LeMme 3.6. Si $\left(K_{n}\right)_{n \in \omega}$ est une suite de compacts d'un compact métrisable $E$ et $\mathcal{F}$ est de classe de Baire $\Sigma_{\alpha}^{0}$ (resp. $\left.\Pi_{\alpha}^{0}\right)$ dans $2^{\omega}$, alors $H_{\mathcal{F}}\left(K_{n}\right)$ est de classe $\Sigma_{\alpha+1}^{0}\left(\right.$ resp. $\left.\Pi_{\alpha+1}^{0}\right)$ dans $E$.

Démonstration. L'application $\Phi: E \rightarrow 2^{\omega}, x \mapsto\left\{n \in \omega ; x \in K_{n}\right\}$, est de première classe de Baire; en effet, tout ouvert de $2^{\omega}$ est une réunion dénombrable d'ouverts élémentaires de la forme $I_{s}=\left\{A \in 2^{\omega} ; \forall n<\lg (s)\right.$, $A(n)=s(n)\}$ où $s \in 2^{<\omega}$ est une suite finie de longueur $\lg (s)$. Alors

$$
x \in \Phi^{-1}\left(I_{s}\right) \Leftrightarrow\left(\forall n \in s^{-1}(1), x \in K_{n}\right) \text { et }\left(\forall n \in s^{-1}(0), x \notin K_{n}\right),
$$


donc l'image réciproque par $\Phi$ de tout ouvert de $2^{\omega}$ est un $\mathcal{F}_{\sigma}$ de $E \times \mathcal{K}(E)^{\omega}$, car $\{(x, K) ; x \in K\}$ est un fermé de $E \times \mathcal{K}(E)$.

Comme $H_{\mathcal{F}}\left(K_{n}\right)=\Phi^{-1}(\mathcal{F})$, on a bien le résultat souhaité.

Corollaire 3.7. Si $\mathcal{F}$ est un filtre borélien libre, alors l'opération $\mathcal{F}$ n'est pas idempotente (i.e., $\mathcal{F} \prec \mathcal{F}_{2}$ ).

Démonstration. Si $\mathcal{F}$ est idempotente, alors, pour tout ordinal dénombrable $\alpha \geq 1, \mathcal{F} \equiv \mathcal{F}_{\alpha}$. Comme, $\mathcal{F} r \subseteq \mathcal{F}$, on a $\mathcal{F} r_{\alpha} \preceq \mathcal{F}_{\alpha}$, donc $\mathcal{F} r_{\alpha} \preceq \mathcal{F}$.

La base $\mathcal{F}$ est borélienne d'une certaine classe de Baire $\beta+1$. D'après le Lemme 3.6, si $E$ est un compact métrisable, alors $\lim _{\mathcal{F}}\left(\Delta_{1}^{0}\right) \subseteq \Delta_{\beta}^{0}$. Or $2^{\omega}$ est un compact métrisable de dimension 0 , pour lequel on aurait pour tout ordinal dénombrable $\alpha \geq 1$,

$$
\Delta_{\alpha+1}^{0}=\lim _{\mathcal{F} r_{\alpha}}\left(\Delta_{1}^{0}\right) \subseteq \lim _{\mathcal{F}}\left(\Delta_{1}^{0}\right) \subseteq \Delta_{\beta+1}^{0},
$$

ce qui contredirait le théorème de Borel-Lebesgue.

ThÉORÈme 3.8. Si $\mathcal{F}$ est un filtre borélien libre, la suite $\left(\mathcal{F}_{\alpha}\right)_{\alpha<\omega_{1}}$ est strictement croissante pour $\prec\left(\right.$ i.e., $\left.\operatorname{ht}(\mathcal{F})=\omega_{1}\right)$.

Démonstration. Sinon, il existe un ordinal dénombrable $\alpha \geq 1$ tel que $\mathcal{F}_{\alpha} \equiv \mathcal{F}_{\alpha+1}$. Alors, pour tout ordinal dénombrable $\beta>\alpha, \mathcal{F}_{\beta} \equiv \mathcal{F}_{\alpha}$, donc l'opération $\mathcal{F}_{\alpha}$ est idempotente, ce qui est impossible, car $\mathcal{F}_{\alpha}$ est un filtre borélien libre.

Remarque. Rappelons la méthode de B. Aniszczyk, J. Burzyk et A. Kamiński qui utilise également le théorème de Borel-Lebesgue : le stabilisé par réunion croissante de la classe

$$
\mathcal{M}=\left\{(A, B) \in 2^{\omega} \times 2^{\omega} ; \exists C \in \Delta_{1}^{0}, A \subset C \subset B^{c}\right\}
$$

ne peut être obtenu par moins de $\omega_{1}$ opérations.

Par des arguments du genre de ceux du Lemme 3.3, on peut montrer que $\left\{(A, B) \in 2^{\omega} \times 2^{\omega} ; \exists C \in \Delta_{1+\alpha}^{0}, A \subset C \subset B^{c}\right\} \subset \mathcal{M}^{\alpha \uparrow}$ et $\mathcal{M}^{\alpha \uparrow} \subset$ $\left\{(A, B) \in 2^{\omega} \times 2^{\omega} ; \exists C \in \Sigma_{1+\alpha}^{0}, A \subset C \subset B^{c}\right.$ et $\left.\exists D \in \Pi_{1+\alpha}^{0}, A \subset D \subset B^{c}\right\}$, ce qui permet de conclure immédiatement.

\section{Un théorème de hiérarchie pour la réunion croissante com- pacte}

a) Les résultats. Pour étudier les classes de compacts nous aurons besoin d'une version plus fine du Corollaire 3.5.

ThÉORÈme 4.1. Pour tout couple d'ordinaux $(\alpha, \beta)$ avec $1 \leq \alpha<\beta<\omega_{1}$, on $a$

$$
\mathcal{P}_{\beta} \npreceq \mathcal{F} r_{\alpha}
$$


La démonstration de ce résultat constitue l'élément principal de cet article et sera développée dans les sous-sections b), c) et d).

Dans les questions de stabilité, les compacts jouent un rôle particulier. Par exemple, N. Bari a montré que la réunion dénombrable de compacts d'unicité est un ensemble d'unicité, alors que le problème reste ouvert dans le cas général (même pour la réunion de deux $\mathcal{G}_{\delta}$ d'unicité). D'autre part, une réunion croissante de compacts de convergence absolue est un ensemble de convergence absolue [7], mais c'est faux pour des ensembles non compacts : c'est ce problème qui a motivé notre étude.

Dans la suite, $\mathcal{C}$ désignera toujours une classe héréditaire de compacts d'un espace métrisable compact $E$ et $\mathcal{F}$ une base monotone sur $\omega$.

DÉFINITION 4.2. On pose

$$
\mathcal{C}_{K}^{\mathcal{F}}=\mathcal{C}^{\mathcal{F}} \cap \mathcal{K}(E)=\left\{K \in \mathcal{K}(E) ; \exists\left(K_{n}\right)_{n \in \omega} \subseteq \mathcal{C}, K=H_{\mathcal{F}}\left(K_{n}\right)\right\} .
$$

On en déduit, comme dans la Définition 2.1, les notations $\mathcal{C}_{K}^{\alpha \mathcal{F}}\left(\alpha<\omega_{1}\right)$, $\mathcal{C}_{K}^{\infty \mathcal{F}}, \operatorname{rg}_{\mathcal{F}, K}(\mathcal{C})$ et $\operatorname{ht}(\mathcal{F}, K)$.

Reprenons les notations du Paragraphe 1.b, en remarquant que les $I_{n}=$ $\left\{A \in 2^{\omega} ; n \in A\right\}(n \in \omega)$ sont compacts, et posons

$$
I=\mathcal{I} \cap \mathcal{K}\left(2^{\omega}\right)=\bigcup_{n \in \omega} \mathcal{K}\left(I_{n}\right)
$$

Remarquons que $I$ est un ouvert de $\mathcal{K}\left(2^{\omega}\right)$.

Attention, $I$ ne joue pas a priori le rôle d'exemple canonique dans l'étude des opérations $(\mathcal{F}, K)$ et on a aucun équivalent de la Proposition 1.5.

Nous allons étudier en détail le cas de l'opération de réunion croissante. Nous noterons $\mathcal{C}_{K}^{\alpha \uparrow}=\mathcal{C}_{K}^{\alpha \mathcal{F} r}\left(\alpha<\omega_{1}\right.$ ou $\left.\alpha=\infty\right), \uparrow K(\mathcal{C})=\operatorname{rg}_{\mathcal{F} r, K}(\mathcal{C})$ et $\uparrow(\mathcal{C})=\operatorname{rg}_{\mathcal{F} r}(\mathcal{C})$.

Remarque. $\mathcal{C}^{\alpha \mathcal{P}} \subseteq \mathcal{C}_{K}^{\alpha \uparrow}$.

En effet, d'après la Proposition $1.3, \mathcal{C}_{K}^{\mathcal{P}}=\mathcal{C}^{\mathcal{P}}$, donc $\mathcal{C}^{\alpha \mathcal{P}}=\mathcal{C}_{K}^{\alpha \mathcal{P}} \subseteq \mathcal{C}_{K}^{\alpha \uparrow}$.

Proposition 4.3. $\uparrow K(I)=\uparrow(I)=\omega_{1}$. En particulier, ht $(\uparrow, K)=\omega_{1}$.

Démonstration. Il suffit de montrer que pour tout couple $(\alpha, \beta)$ d'ordinaux avec $1 \leq \alpha<\beta<\omega_{1}$, on a $I_{K}^{\beta \uparrow} \backslash \mathcal{I}^{\alpha \uparrow} \neq \emptyset$.

Notons que $\mathcal{P}_{\beta}=H_{\mathcal{P}_{\beta}}\left(I_{n}\right) \in I_{K}^{\beta \uparrow}$ (remarque précédente). Supposons que $\mathcal{P}_{\beta} \in \mathcal{I}^{\alpha \uparrow}$; alors il existe une suite $\left(X_{k}\right)_{k \in \omega} \subset \mathcal{I}$ telle que $\mathcal{P}_{\beta}=H_{\mathcal{F} r_{\alpha}}\left(X_{k}\right)$. Mais par définition de $\mathcal{I}$, pour tout $k \in \omega$, il existe un entier $n_{k}$ tel que $X_{k} \subseteq I_{n_{k}}$, donc $\mathcal{P}_{\beta} \subseteq H_{\mathcal{F} r_{\alpha}}\left(I_{n_{k}}\right)$.

Considérons $\varphi: \omega \rightarrow \omega, k \mapsto n_{k}$. Pour tout $A \in \mathcal{P}_{\beta}$, on a $A \in H_{\mathcal{F} r_{\alpha}}\left(I_{n_{k}}\right)$, donc $\mathcal{F} r_{\alpha} \ni\left\{k ; A \in I_{n_{k}}\right\}=\left\{k ; n_{k} \in A\right\}=\varphi^{-1}(A)$, ce qui revient à dire que $\mathcal{P}_{\beta} \preceq \mathcal{N}_{\alpha}$ et qui contredit le Théorème 4.1. 
La même méthode permet de montrer que diverses classes de compacts liées à la classe des compacts de convergence absolue sont de rang $\uparrow K$ égal à $\omega_{1}([6],[7])$.

Corollaire 4.4. Pour tout ordinal dénombrable $\alpha \geq 1$, il existe une sous-classe $\mathcal{C}$ borélienne $\left(\mathcal{K}_{\sigma}\right)$ de $\mathcal{K}\left(2^{\omega}\right)$ telle que $\uparrow K(\mathcal{C})=\uparrow(\mathcal{C})=\alpha$.

Démonstration. Soit $K \in I_{K}^{\alpha \uparrow} \backslash I_{K}^{<\alpha \uparrow}$ (donné par la proposition précédente). Par définition de $I_{K}^{\alpha \uparrow}$ et comme $I$ est héréditaire dans $\mathcal{K}\left(2^{\omega}\right)$, il existe une suite $\left(K_{n}\right)_{n \in \omega}$ d'éléments de $I \cap \mathcal{K}(K)$ telle que $K=H_{\mathcal{P}_{\alpha}}\left(K_{n}\right)$ et qu'à toutes les étapes de l'itération de l'opération $\mathcal{P}$ les ensembles obtenus soient des compacts. Posons $\mathcal{C}=\bigcup_{n \in \omega} \mathcal{K}\left(K_{n}\right)$. Alors, $\mathcal{C}$ est un $\mathcal{K}_{\sigma}$ de $\mathcal{K}\left(2^{\omega}\right)$ et $\uparrow K(\mathcal{C})=\uparrow(\mathcal{C})=\alpha$; cette dernière affirmation se démontre comme dans la Proposition 2.7.

Un exemple particulièrement intéressant est celui de la classe $N$ des compacts de convergence absolue qui est une $\uparrow$-classe de compacts (i.e., $\uparrow K(N)=0)$ telle que $\uparrow(N)=\omega_{1}([5],[7])$. C'est également le cas de la classe $M=\mathcal{M} \cap\left(\mathcal{K}\left(2^{\omega}\right) \times \mathcal{K}\left(2^{\omega}\right)\right)$ où $\mathcal{M}$ est la classe introduite par B. Aniszczyk, J. Burzyk et A. Kamiński.

Ces résultats nous amènent à poser la question suivante.

Question. Quels sont les couples $(\alpha, \beta)$ d'ordinaux $\leq \omega_{1}$ tels qu'il existe une classe $\mathcal{C}$ héréditaire (borélienne?) de compacts vérifiant $\uparrow K(\mathcal{C})=\alpha$ et $\uparrow(\mathcal{C})=\beta$ ?

Le problème pour $\alpha>\beta$ ne se pose que si $\beta \geq 2$ et si la réponse à la question suivante est positive.

Question. Existe-t-il une classe $\mathcal{C}$ héréditaire (borélienne?) de compacts de $E$ telle que $\mathcal{C}_{K}^{2 \uparrow} \neq \mathcal{C}^{2 \uparrow} \cap \mathcal{K}(E)$ ? Qu'en est-il de la classe $I$ en particulier?

b) La propriété de séparation. La propriété étudiée ici est essentielle dans la démonstration du Théorème 4.1.

DÉfinition 4.5. Soient $\mathcal{F}$ une base monotone sur $\omega, A \in \widehat{\mathcal{F}}$ et $f$ : $A \rightarrow \omega$. Considérons le jeu $J(\mathcal{F}, A, f)$ infini entièrement déterminé à deux joueurs I et II. C'est II qui commence.

$$
\begin{array}{cc}
\text { I } & \text { II } \\
& n_{0} \\
n_{1} \geq n_{0} & \\
\vdots & n_{2} \geq n_{1}
\end{array}
$$


I et II jouent chacun leur tour un entier supérieur au précédent joué. I gagne la partie si

$$
f^{-1}\left(\bigcup _ { i \in \omega } \left[n_{2 i}, n_{2 i+1}[) \in \widehat{\mathcal{F}} .\right.\right.
$$

On dira que $\mathcal{F}$ a la propriété de séparation si pour tous $A \in \widehat{\mathcal{F}}$ et $f: A \rightarrow$ $\omega$ n'ayant aucune fibre $\widehat{\mathcal{F}}$, I a une stratégie gagnante dans le jeu $J(\mathcal{F}, A, f)$ (où une fibre est l'image réciproque d'un singleton).

Le choix du terme "séparation" se justifie par la proposition suivante.

Proposition 4.6. Soit $\left(\mathcal{F}_{i}, A_{i}, f_{i}\right)_{i \in \omega}$ une famille de triplets comme cidessus où I a une stratégie gagnante dans $J\left(\mathcal{F}_{i}, A_{i}, f_{i}\right)$. Alors, pour tout $i \in$ $\omega$, il existe une partie $A_{i}^{\prime} \in \widehat{\mathcal{F}}_{i}$ incluse dans $A_{i}$ telle que $f_{i}\left(A_{i}^{\prime}\right) \cap f_{j}\left(A_{j}^{\prime}\right)=\emptyset$ pour $i \neq j$.

Démonstration. Nous allons faire jouer les différentes stratégies de I dans les jeux $J\left(\mathcal{F}_{i}, A_{i}, f_{i}\right)$ à tour de rôle dans une même partie. Soit $\varphi: \omega \rightarrow \omega$ une application infinijective (i.e., $\forall n \in \omega, \varphi^{-1}\{n\}$ infini).

Soit $i \in \omega$; supposons les $2 i+1$ premiers coups $\left(n_{0}, \ldots, n_{2 i}\right)$ joués. Posons $j=\varphi(i) ; \varphi^{-1}\{j\} \cap\left[0, i\left[=\left\{i_{0}, \ldots, i_{k-1}\right\}\right.\right.$ avec $i_{0}<i_{1}<\ldots<i_{k-1}<i$. Alors l'entier $n_{2 i+1}$ sera donné par la stratégie de I dans le jeu $J\left(\mathcal{F}_{i}, A_{i}, f_{i}\right)$ où ont été joués les $2 k+1$ coups $\left(n_{2 i_{0}}, n_{2 i_{0}+1}, n_{2 i_{1}}, \ldots, n_{2 i_{k-1}}, n_{2 i_{k-1}+1}, n_{2 i}\right)$.

Posons, pour tout $j \in \omega, A_{j}^{\prime}=f_{j}^{-1}\left(\bigcup_{i \in \varphi^{-1}\{j\}}\left[n_{2 i}, n_{2 i+1}[)\right.\right.$; par construction $A_{j}^{\prime} \in \widehat{\mathcal{F}}_{j}$ et les $f_{j}\left(A_{j}^{\prime}\right)$ sont deux à deux disjoints.

Lemme 4.7. Si $\mathcal{F}$ est un filtre sur $\omega, A \in \widehat{\mathcal{F}}$ et $f: A \rightarrow \omega$ sans fibre $\widehat{\mathcal{F}}$, alors le fait que I ou II commence dans le jeu $J(\mathcal{F}, A, f)$ n'a pas d'importance.

Dé monstration. Pour tout $n \in \omega, f^{-1}[0, n[\notin \widehat{\mathcal{F}}$, car $\widehat{\mathcal{F}}$ est une grille et $f$ est sans fibre $\widehat{\mathcal{F}}$, donc l'expression " $f^{-1}\left(\bigcup_{i \in \omega}\left[n_{2 i}, n_{2 i+1}[) \in \widehat{\mathcal{F}}\right.\right.$ " ne dépend pas des premières valeurs $n_{i}(i \in \omega)$.

Proposition 4.8. Si $\mathcal{F}$ est un filtre borélien, alors $\mathcal{F}$ a la propriété de séparation.

Démonstration. Raisonnons par l'absurde et supposons qu'il existe $A \in \widehat{\mathcal{F}}$ et $f: A \rightarrow \omega$ sans fibre $\widehat{\mathcal{F}}$ tels que I n'ait pas de stratégie gagnante dans le jeu $J(\mathcal{F}, A, f)$.

L'ensemble $\left\{\left(n_{i}\right)_{i \in \omega} \in \omega^{\omega} ; \forall i \in \omega, n_{i} \leq n_{i+1}\right.$ et $f^{-1}\left(\bigcup_{i \in \omega}\left[n_{2 i}, n_{2 i+1}[)\right.\right.$ $\in \widehat{\mathcal{F}}\}$ est borélien dans $\omega^{\omega}$ muni de la topologie produit; en effet, l'application $\Phi=f^{-1}: 2^{\omega} \rightarrow \omega^{\omega}, A \mapsto f^{-1} A$, est continue et l'application $\Psi: \omega^{\omega} \rightarrow$ $2^{\omega},\left(n_{i}\right)_{i \in \omega} \mapsto \bigcup_{i \in \omega}\left[n_{2 i}, n_{2 i+1}[\right.$, est de première classe, donc la condition " $f^{-1}\left(\bigcup_{i \in \omega}\left[n_{2 i}, n_{2 i+1}[) \in \widehat{\mathcal{F}} "\right.\right.$ est équivalente à " $\left(n_{i}\right)_{i \in \omega} \in(\Phi \circ \Psi)^{-1} \widehat{\mathcal{F}}$ " et elle est borélienne dès que $\mathcal{F}$ est borélienne. 
Alors le jeu $J(\mathcal{F}, A, f)$ est déterminé (théorème de $\mathrm{D}$. A. Martin), c'està-dire que l'un des deux joueurs possède une stratégie gagnante. D'après notre hypothèse, II possède une stratégie gagnante dans le jeu $J(\mathcal{F}, A, f)$, ou ce qui revient au même dans le jeu $J^{\prime}(\mathcal{F}, A, f)$, jeu similaire à $J(\mathcal{F}, A, f)$ où I commence (Lemme 4.7).

Nous ferons jouer I dans le jeu $J(\mathcal{F}, A, f)$ suivant la stratégie de II dans le jeu $J^{\prime}(\mathcal{F}, A, f)$. Mais comme II gagne dans le jeu $J^{\prime}(\mathcal{F}, A, f)$, il en résulte que $f^{-1}\left(\bigcup_{i \in \omega}\left[n_{2 i+1}, n_{2 i+2}[) \notin \widehat{\mathcal{F}}\right.\right.$, donc $f^{-1}\left(\bigcup_{i \in \omega}\left[n_{2 i}, n_{2 i+1}[)\right.\right.$ $=A \cap\left(f^{-1}\left(\bigcup_{i \in \omega}\left[n_{2 i+1}, n_{2 i+2}[)\right)^{c} \in \mathcal{F} \subseteq \widehat{\mathcal{F}}\right.\right.$; nous avons exhibé une stratégie gagnante pour I dans le jeu $J(\mathcal{F}, A, f)$.

Remarque. Si $\mathcal{F}$ est un ultrafiltre, alors $\mathcal{F}$ n'a pas la propriété de séparation.

En effet, le jeu $J(\mathcal{F}, A, f)$ n'est pas déterminé, car $\widehat{\mathcal{F}}=\mathcal{F}$, donc une stratégie gagnante pour I permet de construire une stratégie gagnante pour II et vice-versa.

c) Démonstration du Théorème 4.1

Notations. 1) On dira que I passe au $i$-ième coup (dans un jeu $G(\ldots)$ ), si I joue $B_{i}=\omega$. Dans ce cas, II peut répondre ce qu'il veut.

2) Si $\mathcal{F}$ est une base monotone sur $\omega$ et $A \in \mathcal{F}$, nous noterons $\mathcal{F}(A)$ l'ensemble des parties appartenant à $\mathcal{F}$ et incluses dans $A$.

3) Notons la propriété élémentaire suivante : pour tout $A \in \widehat{\mathcal{F} r}$ et toute application $f: A \rightarrow \omega$ sans fibre $\widehat{\mathcal{F} r}$, il existe $A^{\prime} \in \widehat{\mathcal{F} r}(A)$ tel que $f: A^{\prime} \rightarrow \omega$ soit injective. Nous dirons que $\mathcal{P}$ est lié à $\mathcal{F} r$.

Démonstration $\mathrm{d} u$ Théorème 4.1. Nous allons montrer par récurrence sur $\alpha$ que pour tout $A \in \widehat{\mathcal{F}}_{\alpha}$, tout ordinal $\beta$ avec $\alpha<\beta<\omega_{1}$, et toute application $\varphi: A \rightarrow \widetilde{T}_{\beta}$, il existe une partie $A^{\prime} \in \widehat{\mathcal{F}}_{\alpha}$ incluse dans $A$ telle que $\varphi\left(A^{\prime}\right)^{c} \in \mathcal{P}_{\beta}$, c'est-à-dire que I est une stratégie gagnante dans le jeu $G\left(\mathcal{P}, \beta, \varphi\left(A^{\prime}\right)^{c}\right)$.

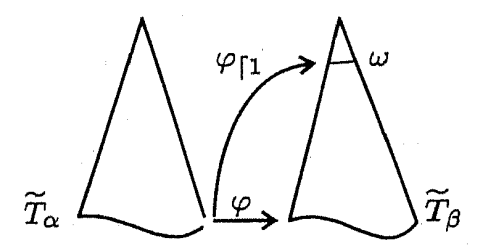

On note $\varphi_{\lceil i}$ l'application de $A$ dans $\omega$ qui à $u \in A$ associe le $i$-ième terme de la suite $\varphi(u) \in T_{\beta}$. S'il existe $A^{\prime} \in \widehat{\mathcal{F}}_{\alpha}(A)$ telle que $\varphi_{\lceil 1}\left(A^{\prime}\right)$ soit un singleton, alors I a une stratégie gagnante dans le jeu $G\left(\mathcal{P}, \beta, \varphi\left(A^{\prime}\right)^{c}\right)$ en jouant $\varphi_{\lceil 1}\left(A^{\prime}\right)^{c} \in \mathcal{P}$ au premier coup et en passant ensuite. Sinon : 
FAIT 4.9. Si pour tout $A^{\prime} \in \widehat{\mathcal{F}}_{\alpha}(A)$, on a $\varphi_{\lceil 1}\left(A^{\prime}\right)$ infini, alors il existe une partie $A^{\prime} \in \widehat{\mathcal{F}}_{\alpha}(A)$ telle que I ait une stratégie gagnante dans le jeu $G\left(\mathcal{P}, \beta, \varphi\left(A^{\prime}\right)^{c}\right)$ en passant au premier coup.

Nous allons démontrer le fait par récurrence sur $\alpha \geq 1$.

Cas $\alpha=1$. Comme $\mathcal{P}$ est lié à $\mathcal{F} r$, il existe une partie $A^{\prime} \in \widehat{\mathcal{F} r}$ incluse dans $A$, telle que $\varphi: A^{\prime} \rightarrow \omega$ soit injective. Alors I a une stratégie gagnante dans le jeu $G\left(\mathcal{P}, \beta, \varphi\left(A^{\prime}\right)^{c}\right)$ en passant au premier coup; en effet, si $n_{0}$ est le premier coup de II, alors I répondra, pour son deuxième coup, $\operatorname{par}\left(\varphi_{\lceil 2}\left[\varphi_{\lceil 1}{ }^{-1}\left\{n_{0}\right\}\right]\right)^{c} \in \mathcal{P}$ et passera ensuite.

Cas général. Supposons l'hypothèse de récurrence vérifiée jusqu'au $\operatorname{rang} \alpha(\alpha \geq 2)$.

Considérons, pour tout $j \in \omega$, l'application $f_{j}: A_{j} \rightarrow \omega, u \mapsto \varphi_{\lceil 1}\left(j^{\wedge} u\right)$. Posons $J=\left\{j \in \omega ; f_{j}\right.$ a une fibre $\left.\widehat{\mathcal{F}}_{[\alpha]_{j}}\right\}$. Deux cas sont à envisager.

1 er cas : $\widetilde{A}^{\prime}=\widetilde{A} \cap J \in \widehat{\mathcal{F} r}$ (i.e., est infini). Pour tout $j \in \widetilde{A}^{\prime}$, il existe $A_{j}^{\prime} \in \widehat{\mathcal{F}}_{[\alpha]_{j}}$ incluse dans $A_{j}$ telle que $\varphi_{\lceil 1}\left(\{j\} \times A_{j}^{\prime}\right)$ est un singleton noté $\left\{k_{j}\right\}$. Considérons l'application $f: \widetilde{A}^{\prime} \rightarrow \omega, j \mapsto k_{j}$; pour toutes parties $\widetilde{A}^{\prime \prime} \in \widehat{\mathcal{F} r}\left(\widetilde{A}^{\prime}\right)$, on a $f\left(\widetilde{A}^{\prime \prime}\right) \in \widehat{\mathcal{P}}$, sinon il existe $A^{\prime} \in \widehat{\mathcal{F}}_{\alpha}(A)$ telle que $\varphi_{\lceil 1}\left(A^{\prime}\right)$ soit un singleton, ce qui est contraire à l'hypothèse du fait. Comme $\mathcal{P}$ est lié à $\mathcal{F} r$, il existe une partie $\widetilde{A}^{\prime \prime} \in \widehat{\mathcal{F} r}\left(\widetilde{A}^{\prime}\right)$ telle que $f: \widetilde{A}^{\prime \prime} \rightarrow \omega, j \mapsto k_{j}$, soit injective.

De plus $\alpha<\beta$, donc il existe un $n \in \omega$ tel que $[\beta]_{n} \geq \alpha$; mais $f^{-1}[0, n[$ est fini, donc, quitte à remplacer $\widetilde{A}^{\prime \prime}$ par $\widetilde{A}^{\prime \prime} \backslash f^{-1}[0, n[\in \widehat{\mathcal{F} r}$, on a pour tout $j \in \widetilde{A}^{\prime \prime},[\beta]_{k_{j}} \geq \alpha$. Considérons, pour tout $j \in \widetilde{A}^{\prime \prime}$, l'application $\varphi_{j}: A_{j}^{\prime} \rightarrow$ $T_{[\beta]_{k_{j}}}$ définie par, pour tout $u \in A_{j}^{\prime}, \varphi\left(j^{\wedge} u\right)=k_{j} \wedge \varphi_{j}(u)$; par hypothèse de récurrence appliquée à $[\alpha]_{j}\left(<[\beta]_{k_{j}}\right)$, il existe une partie $A_{j}^{\prime \prime} \in \widehat{\mathcal{F}}_{[\alpha]_{j}}$ incluse dans $A_{j}^{\prime}$ telle que $\varphi\left(A_{j}^{\prime \prime}\right)^{c} \in \mathcal{P}_{[\beta]_{k_{j}}}$.

Posons $A^{\prime}=\left\{j^{\wedge} u ; j \in \widetilde{A}^{\prime \prime}, u \in A_{j}^{\prime \prime}\right\}$. En appliquant l'hypothèse de récurrence, on construit comme précédemment une partie $A^{\prime}=\left\{j^{\wedge} u ; j \in\right.$ $\left.\widetilde{A}^{\prime \prime}, u \in A_{j}^{\prime \prime}\right\}$. Alors $A^{\prime} \in \widehat{\mathcal{F}}_{\alpha}(A)$ et I a une stratégie gagnante dans le jeu $G\left(\mathcal{P}, \beta, \varphi\left(A^{\prime}\right)^{c}\right)$ en passant au premier coup; en effet, si $n_{0}$ est le premier coup de II, alors il existe au plus un $k_{j}$ égal à $n_{0}$. Si $k_{j}=n_{0}$, I jouera à partir du deuxième coup avec la stratégie de I dans le jeu $G\left(\mathcal{P}, \beta, \varphi_{j}\left(A_{j}^{\prime \prime}\right)^{c}\right)$, sinon I passera tout le temps.

2 ème cas : $\widetilde{A}^{\prime}=\widetilde{A} \backslash J \in \widehat{\mathcal{F} r}$. Le deuxième cas semble plus difficile car les $f_{j}\left(A_{j}\right)$ ne sont plus des singletons, mais ces ensembles peuvent être rendus disjoints à l'aide du lemme de séparation.

En effet, $\mathcal{F} r$ est un filtre borélien, donc tous les $\mathcal{F} r_{\alpha}$ sont des filtres boréliens : on en déduit que, pour tout $j \in \widetilde{A}^{\prime}$, il existe $A_{j}^{\prime} \in \widehat{\mathcal{G}}_{[\alpha]_{j}}$ inclus 
dans $A_{j}$ tel que les $f_{i}\left(A_{i}^{\prime}\right), i \in \widetilde{A}^{\prime}$, soient deux à deux disjoints (propriété de séparation).

Considérons, pour tout $j \in \widetilde{A}^{\prime \prime}$, l'application $\varphi_{j}: A_{j}^{\prime} \rightarrow T_{\beta}, u \mapsto \varphi\left(j^{\wedge} u\right)$; on a $\varphi_{j\lceil 1}=f_{j}$. Alors pour tout $B_{j} \in \widehat{\mathcal{F}}_{[\alpha]_{j}}\left(A_{j}^{\prime}\right), f_{j}\left(B_{j}\right)$ est infini, ce qui permet d'utiliser l'hypothèse de récurrence (du fait) au rang $[\alpha]_{j}$ : il existe une partie $A_{j}^{\prime \prime} \in \widehat{\mathcal{F}}_{[\alpha]_{j}}\left(A_{j}^{\prime}\right)$ telle que I ait une stratégie gagnante dans le jeu $G\left(\mathcal{P}, \beta, \varphi_{j}\left(A_{j}^{\prime \prime}\right)^{c}\right)$ en passant au premier coup. Posons $A^{\prime}=\left\{j^{\wedge} u ; j \in \widetilde{A}^{\prime \prime}\right.$, $\left.u \in A_{j}^{\prime \prime}\right\}$. Alors, $A^{\prime} \in \widehat{\mathcal{F}}_{\alpha}(A)$ et I a une stratégie gagnante dans le jeu $G\left(\mathcal{P}, \beta, \varphi\left(A^{\prime}\right)^{c}\right)$ en passant au premier coup; en effet, si $n_{0}$ est le premier coup de II, alors I jouera pour son deuxième coup dans le jeu $G\left(\mathcal{P}, \beta, \varphi\left(A^{\prime}\right)^{c}\right)$ le deuxième coup donné par la stratégie de I dans le jeu $G\left(\mathcal{P}, \beta, \varphi_{j}\left(A_{j}^{\prime \prime}\right)^{c}\right)$ à partir du deuxième coup (puisque I a passé au premier coup dans ce jeu) où $j$ est l'unique valeur pour laquelle $n_{0} \in f_{j}\left(A_{j}^{\prime \prime}\right)$, et ainsi de suite. Si $n_{0}$ n'appartient à aucun $f_{j}\left(A_{j}^{\prime \prime}\right)$, il suffira à I de passer jusqu'à la fin pour gagner.

C'est l'obligation de jouer un deuxième coup dans la première étape de la récurrence qui nécessite l'intervention du fait dans la démonstration. On conclut comme dans le premier cas et ceci termine la démonstration du théorème.

d) Démonstration courte d'un Théorème 4.1 simplifié

Proposition 4.10. Pour tout entier $n$, on a $\mathcal{P}_{n+1} \npreceq \mathcal{F} r_{n}$.

Lemme 4.11. Soit $\mathcal{F}$ et $\mathcal{G}$ deux bases monotones sur $\omega$. Alors

$$
\mathcal{F} \npreceq \mathcal{G} \Rightarrow \mathcal{F} \otimes \mathcal{P} \npreceq \mathcal{G} \otimes \mathcal{F} r .
$$

Démonstration. Soit $\varphi: \omega \rightarrow \omega$. Montrons qu'il existe une partie $A \in \mathcal{G} \widehat{\otimes \mathcal{F} r}$ telle que $\varphi(A)^{c} \in \mathcal{F} \otimes \mathcal{P}$. Rappelons que $\mathcal{G} \widehat{\otimes \mathcal{F} r}=\widehat{\mathcal{G}} \otimes \widehat{\mathcal{F} r}$. Soit $\varphi_{i}: \omega \rightarrow \omega, j \mapsto \varphi(\langle i, j\rangle)_{\lceil 1}$, où $n=\left\langle n_{\lceil 1}, n_{\lceil 2}\right\rangle$ pour tout $n \in \omega$, et

$$
J=\left\{i \in \omega ; \exists A_{i} \in \widehat{\mathcal{F} r}, \exists k_{i} \in \omega, \varphi_{i}\left(A_{i}\right)=\left\{k_{i}\right\}\right\} .
$$

Soit $f: \omega \rightarrow \omega$ la fonction qui associe $k_{i}$ à $i \in J$ et rien sinon. Comme $\mathcal{F} \npreceq \mathcal{G}$ il existe $\widetilde{A} \in \widehat{\mathcal{G}}$ tel que $f(\widetilde{A})^{c} \in \mathcal{F}$. D'autre part il existe des parties $A_{i}$ pour tout $i \notin J$ telles que les $\varphi_{i}\left(A_{i}\right)$ soient deux à deux disjoints et les applications $\varphi_{i}: A_{i} \rightarrow \omega$ soient injectives pour chaque $i \notin J$ (en utilisant la propriété de séparation et le fait que $\mathcal{P}$ soit lié à $\mathcal{F} r)$. Alors $\bigcup_{i \notin J} \varphi_{i}\left(A_{i}\right) \cap\langle k,\rangle.(\omega)$ est de cardinal $\leq 1$ pour tout $k \in \omega$. Donc $A=\langle\rangle \bigcup_{i \in \widetilde{A}}\{i\} \times A_{i}$ appartient à $\widehat{\mathcal{G}} \otimes \widehat{\mathcal{F} r}$ et $\varphi(A)^{c}$ appartient à $\mathcal{F} \otimes \mathcal{P}$.

Démonstration de la Proposition 4.10. Puisque $\mathcal{P} \npreceq \mathcal{U}_{1}$, $\mathcal{P}_{2}=(\mathcal{P} \otimes \mathcal{P}) \npreceq\left(\mathcal{U}_{1} \otimes \mathcal{F} r\right)=\mathcal{F} r$. Le résultat s'obtient par récurrence sur $n$ : si $\mathcal{P}_{n} \npreceq \mathcal{F} r_{n-1}$, alors $\mathcal{P}_{n+1}=\mathcal{P}_{n} \otimes \mathcal{P} \npreceq \mathcal{F} r_{n-1} \otimes \mathcal{F} r=\mathcal{F} r_{n}$. 
5. Un théorème de hiérarchie pour la réunion asymptotique. Cette section est plus exotique que les précédentes. Son but est d'obtenir d'autres informations sur le préordre $\preceq$, d'autres théorèmes de hiérarchie liant deux bases monotones et d'étudier plus particulièrement le filtre $\mathcal{A} s$ qui joue un rôle important en Analyse Harmonique dans l'étude des classes de type $\mathcal{W C}$ (Proposition 1.4).

a) Un théorème de hiérarchie

ThÉonème 5.1. Pour tout couple d'ordinaux $(\alpha, \beta)$ avec $1 \leq \alpha<\beta<\omega_{1}$, on a $\mathcal{F} r_{\beta} \npreceq \mathcal{A} s_{\alpha}$.

Lemme 5.2. Pour tout $A \in \widehat{\mathcal{A} s}$ et toute application $f: A \rightarrow \omega$ sans fibre $\widehat{\mathcal{A s}}$, il existe $A^{\prime} \in \widehat{\mathcal{A} s}(A)$ tel que $f: A^{\prime} \rightarrow \omega$ soit finijective (i.e., les fibres de $f$ sont finies). On dira que $\mathcal{F} r$ est lié à $\mathcal{A} s$.

Démonstration. Soit $A \in \widehat{\mathcal{A} s}$ et $f: A \rightarrow \omega$ sans fibre $\widehat{\mathcal{A} s}$. Nous allons construire par récurrence sur $i \in \omega$, des suites $\left(a_{i}\right)_{i \in \omega}$ et $\left(n_{i}\right)_{i \in \omega}$ d'entiers telles que

$$
\begin{gathered}
\forall i \in \omega, \quad f\left[0, a_{i}\left[\subseteq \left[0, n_{i}[\right.\right.\right. \\
\text { et } \quad A^{\prime}=\bigcup_{i \in \omega}\left(\left(\left[0, a_{i+1}[\cap A) \backslash f^{-1}\left[0, n_{i}[]\right) \widehat{\mathcal{A} s} .\right.\right.\right.
\end{gathered}
$$

Supposons $a_{i}$ et $n_{i}$ construits; la densité de $f^{-1}\left[0, n_{i}\right.$ [ est nulle, car $f$ n'a pas de fibre $\widehat{\mathcal{A s}}$, donc il existe $a_{i+1} \in \omega$ tel que

$$
\operatorname{card}\left(\left(\left[0, a_{i+1}[\cap A) \backslash f^{-1}\left[0, n_{i}[) \geq \frac{\mathrm{ds}(A)}{2} \cdot a_{i+1} .\right.\right.\right.\right.
$$

Nous prendrons $n_{i+1}$ tel que $f\left(A \cap\left[0, a_{i+1}[) \subseteq\left[0, n_{i+1}[\right.\right.\right.$.

On vérifie aisément que $\operatorname{ds}\left(A^{\prime}\right) \geq \frac{1}{2} \mathrm{ds}(A)$, donc $A^{\prime} \in \widehat{\mathcal{A s}}$. De plus, $f: A^{\prime} \rightarrow \omega$ est finijective; en effet, si $n<n_{i}$, alors $f^{-1}\{n\} \subseteq\left[0, a_{i}[\right.$.

Question. Existe-t-il une propriété de liaison plus générale telle qu'on puisse dire que si $\mathcal{F}$ et $\mathcal{G}$ sont deux filtres boréliens avec $\mathcal{F} \subseteq \mathcal{G}$ (ou $\mathcal{F} \preceq \mathcal{G}$ ) et si $\mathcal{F}$ est lié à $\mathcal{G}$, alors pour tout couple d'ordinaux $(\alpha, \beta)$ avec $1 \leq \alpha<$ $\beta<\omega_{1}$, on ait $\mathcal{F}_{\beta} \npreceq \mathcal{G}_{\alpha}$ ?

Démonstration du Théorème 5.1. Nous allons montrer par récurrence sur $\alpha$ que pour tout $A \in \widehat{\mathcal{A s}}_{\alpha}$, tout ordinal $\beta$ avec $\alpha<\beta<\omega_{1}$, et toute application $\varphi: A \rightarrow \widetilde{T}_{\beta}$, il existe une partie $A^{\prime} \in \widehat{\mathcal{A s}}_{\alpha}$ incluse dans $A$ telle que $\varphi\left(A^{\prime}\right)^{c} \in \mathcal{F} r_{\beta}$, c'est-à-dire que I ait une stratégie gagnante dans le jeu $G\left(\mathcal{F} r, \beta, \varphi\left(A^{\prime}\right)^{c}\right)$.

Le schéma de la démonstration est le même que pour le Théorème 4.1 en remplaçant $\mathcal{P}$ par $\mathcal{F} r$ et $\mathcal{F} r$ par $\mathcal{A} s$. La seule variante se trouve dans le premier cas de la démonstration du fait : comme on a $\mathcal{F} r$ lié à $\mathcal{A} s$ (Lemme 5.2), il existe une partie $\widetilde{A}^{\prime \prime} \in \widehat{\mathcal{G}}\left(\widetilde{A^{\prime}}\right)$ telle que $f: \widetilde{A}^{\prime \prime} \rightarrow \omega, j \mapsto k_{j}$, soit finijective. 
En appliquant l'hypothèse de récurrence, on construit comme précédemment une partie $A^{\prime}=\left\{j^{\wedge} u ; j \in \widetilde{A}^{\prime \prime}, u \in A_{j}^{\prime \prime}\right\}$. Alors $A^{\prime} \in \widehat{\mathcal{A}}_{\alpha}(A)$ et I a une stratégie gagnante dans le jeu $G\left(\mathcal{F} r, \beta, \varphi\left(A^{\prime}\right)^{c}\right)$ en passant au premier coup; en effet, si $n_{0}$ est le premier coup de II, alors I jouera l'intersection sur $j$ avec $k_{j}=n_{0}$ des stratégies de I dans les jeux $G\left(\mathcal{F} r, \beta, \varphi_{j}\left(A_{j}^{\prime \prime}\right)^{c}\right)$; I jouera bien des éléments de $\mathcal{F} r$, car l'intersection est finie (car $f$ est finijective) et $\mathcal{F} r$ est un filtre. C'est pour cette dernière raison qu'on ne peut pas obtenir $\mathcal{P}_{\beta} \npreceq \mathcal{A} s_{\alpha}$ (voir Proposition 5.4).

b) Un résultat de non commutativité

Proposition 5.3. $\mathcal{F} r \otimes \mathcal{A} s \perp \mathcal{A} s \otimes \mathcal{F} r$.

Démonstration. Soit $\varphi: \omega^{2} \rightarrow \omega^{2}$; montrons qu'il existe un $A \in$ $\widehat{\mathcal{A}} s \otimes \widehat{\mathcal{F} r}$ tel que

$$
\varphi(A) \notin \widehat{\mathcal{F} r} \otimes \widehat{\mathcal{A} s} .
$$

Considérons pour tout $n \in \omega, f_{n}: \omega \rightarrow \omega, k \mapsto \varphi_{\lceil 1}(n, k)$.

1 er cas : Il existe un $\widetilde{A} \in \widehat{\mathcal{A} s}$ tel que, pour tout $n \in \widetilde{A}, f_{n}$ a une fibre $A_{n} \in \widehat{\mathcal{F} r}$. Posons $f_{n}\left(A_{n}\right)=\left\{a_{n}\right\}$. Si la fonction $f: \widetilde{A} \rightarrow \omega, n \mapsto a_{n}$, a une fibre $\widetilde{A}^{\prime} \in \widehat{\mathcal{A} s}$, on gagne en prenant $A=\bigcup_{n \in \widetilde{A}^{\prime}}\{n\} \times A_{n}$. Sinon, comme $\mathcal{F} r$ est lié à $\mathcal{A s}$ (Lemme 5.2), il existe $\widetilde{A}^{\prime} \in \widehat{\mathcal{A} s}$ incluse dans $\widetilde{A}$ telle que $f: \widetilde{A}^{\prime} \rightarrow \omega$ soit finijective. D'autre part, $\mathcal{A} s \npreceq \mathcal{F} r$, donc, pour tout $n \in \widetilde{A}^{\prime}$, il existe $A_{n}^{\prime} \in \widehat{\mathcal{F} r}$ incluse dans $A_{n}$ telle que $\varphi_{n}\left(A_{n}^{\prime}\right) \notin \widehat{\mathcal{A}} s$ où $\varphi_{n}: A_{n} \rightarrow \omega$, $k \mapsto \varphi_{\lceil 2}(n, k)$. Alors $A=\bigcup_{n \in \widetilde{A}^{\prime}}\{n\} \times A_{n}^{\prime} \in \widehat{\mathcal{A} s} \otimes \widehat{\mathcal{F} r}$ et $\varphi(A) \notin \widehat{\mathcal{F} r} \otimes \widehat{\mathcal{A} s} ;$ en effet, $\left\{a \in \omega ;(\varphi(A))_{a} \in \widehat{\mathcal{A} s}\right\}=\emptyset$, car $\widehat{\mathcal{A} s}$ est une grille, et, pour tout $a \in \omega,(\varphi(A))_{a}=\bigcup_{n \in f^{-1}\{a\}} \varphi_{n}\left(A_{n}^{\prime}\right)$ avec $f^{-1}\{a\}$ fini.

2 èm e cas : Il existe une partie $\widetilde{A} \in \widehat{\mathcal{A} s}$ telle que, pour tout $n \in \widetilde{A}$, $f_{n}$ n'a pas de fibre $\widehat{\mathcal{F} r}$. Comme $\mathcal{F} r$ a la propriété de séparation, il existe, pour tout $n \in \widetilde{A}$, une partie $A_{n} \in \widehat{\mathcal{F} r}$ telle que $f_{n}\left(A_{n}\right) \cap f_{m}\left(A_{m}\right)=\emptyset$ pour $n \neq m$. En prenant, pour tout $n \in \widetilde{A}, A_{n}^{\prime} \in \widehat{\mathcal{F} r}$ incluse dans $A_{n}$ telle que $\varphi_{\lceil 2}\left(\{n\} \times A_{n}^{\prime}\right) \notin \widehat{\mathcal{A}}$ s et en posant $A=\bigcup_{n \in \widetilde{A}}\{n\} \times A_{n}^{\prime}$, on aura la conclusion désirée.

On a montré que $\mathcal{F} r \otimes \mathcal{A} s \npreceq \mathcal{A} s \otimes \mathcal{F} r$. Inversement, considérons $\varphi: \omega \rightarrow$ $\omega^{2}$ et montrons qu'il existe un $A \in \widehat{\mathcal{F} r} \otimes \widehat{\mathcal{A} s}$ tel que $\varphi(A) \notin \widehat{\mathcal{A} s} \otimes \widehat{\mathcal{F} r}$.

1 er c a s : Il existe un $\widetilde{A} \in \widehat{\mathcal{A} s}$ tel que, pour tout $n \in \widetilde{A}, f_{n}$ a une fibre $A_{n} \in \widehat{\mathcal{F} r}$. Posons $f_{n}\left(A_{n}\right)=\left\{a_{n}\right\}$. Si la fonction $f: \widetilde{A} \rightarrow \omega, n \mapsto a_{n}$, a une fibre $\widehat{\mathcal{F} r}$, c'est évident; sinon, il existe $\widetilde{A}^{\prime} \in \widehat{\mathcal{F} r}$ incluse dans $\widetilde{A}$ telle que $f\left(\widetilde{A^{\prime}}\right) \notin \widehat{\mathcal{A} s}$ et $f: \widetilde{A}^{\prime} \rightarrow \omega$ soit injective. On conclut en prenant $A=\bigcup_{n \in \widetilde{A^{\prime}}}\{n\} \times A_{n}$. 
2 ème cas : Sinon, il existe une partie $\widetilde{A} \in \widehat{\mathcal{F} r}$ telle que, pour tout $n \in \widetilde{A}, f_{n}$ n'a pas de fibre $\widehat{\mathcal{A}} s$. D'après la propriété de séparation pour $\mathcal{A} s$, il existe, pour tout $n \in \widetilde{A}$, un $A_{n} \in \widehat{\mathcal{A} s}$ tel que $f_{n}: A_{n} \rightarrow \omega$ soit finijective et les $f_{n}\left(A_{n}\right), n \in \widetilde{A}$, disjoints deux à deux. La conclusion est immédiate.

Ceci démontre que $\mathcal{A} s \otimes \mathcal{F} r \npreceq \mathcal{F} r \otimes \mathcal{A} s$.

c) Un résultat de non liaison. Dans la section précédente, on a montré que ht $(\uparrow K)=\omega_{1}$ par l'intermédiaire de l'opération $\mathcal{P}$ qui a la particularité de transformer les suites de compacts en compacts. Nous allons voir que la même méthode ne peut être appliquée au calcul de $h t(\mathcal{A} s, K)$. La raison en est que :

Proposition 5.4. Pour tout ordinal dénombrable $\alpha \geq 1$, on a $\mathcal{P}_{\alpha} \preceq \mathcal{A} s$.

Question. Existe-t-il une base monotone $\mathcal{F}$ compacte incluse dans $\mathcal{A s}$ (ou mieux dans $\mathcal{F} r$ ) telle que $\mathcal{F}_{2} \npreceq \mathcal{A} s ?$

Une réponse négative à cette question enlèverait tout espoir de calculer ht $(\mathcal{A} s, K)$ par une méthode similaire au calcul de ht $(\uparrow, K)$.

Nous allons terminer par la démonstration de la Proposition 5.4.

DÉfinition 5.5. On définit sur $2^{\omega}$ un ordre partiel $\leq$ par, pour tout $A, B \in 2^{\omega}$,

$$
A \leq B \Leftrightarrow \forall n \in \omega, \operatorname{card}(A \cap[0, n[) \leq \operatorname{card}(B \cap[0, n[) .
$$

On appelle borne inférieure (pour $\leq$ sur $2^{\omega}$ ) d'une base monotone $\mathcal{F}$, l'ensemble $A \in 2^{\omega}$ défini par

$$
\forall n \in \omega, \quad \operatorname{card}(A \cap[0, n[)=\inf \{\operatorname{card}(B \cap[0, n[) ; B \in \mathcal{F}\} .
$$

On dit qu'une bijection \langle\rangle de $\omega^{2}$ sur $\omega$ est parfaite, si $(\langle n, \cdot\rangle \omega)_{n \in \omega}$ est décroissante pour $\leq$ et les applications $\langle n, \cdot\rangle: \omega \rightarrow \omega$ sont croissantes $(n \in \omega)$.

ExEMPLES. 1) La borne inférieure de $\mathcal{P}$ est $\omega \backslash\{0\}=01^{\omega}$. Si $\mathcal{F}=$ $\left\{1^{n} 0^{n} 1^{\omega} ; n \in \omega\right\}$, alors la borne inférieure de $\mathcal{F}$ est $(01)^{\omega}$, pourtant $\mathcal{F} \equiv \mathcal{P}$.

2) La borne inférieure de $\mathcal{F} r$ est $\emptyset=0^{\omega}$.

3) Si $\mathcal{F}$ est une base monotone compacte $\left(\right.$ dans $\left.2^{\omega}\right)$ contenue dans $\widehat{\mathcal{F} r}$, alors la borne inférieure de $\mathcal{F}$ appartient à $\widehat{\mathcal{F} r}$.

En effet, supposons que la borne inférieure $A$ de $\mathcal{F}$ est finie de cardinal $k$; pour tout $n \in \omega$, il existe une partie $B_{n} \in \mathcal{F}$ telle que card $\left(B_{n} \cap[0, n[)=\right.$ $\operatorname{card}\left(A \cap\left[0, n[) \leq k\right.\right.$. Par compacité, $\left(B_{n}\right)_{n \in \omega}$ a une sous-suite convergente de limite $B \in \mathcal{F}$; mais, pour tout $n \in \omega, \operatorname{card}(B \cap[0, n[) \leq k, \operatorname{donc} \operatorname{card}(B) \leq k$, ce qui n'est pas possible.

Rappelons que $\mathcal{F}_{\alpha}$ est construit sur $\widetilde{T}_{\alpha}$ et que $\mathcal{F}_{\alpha}$ est envoyée sur $\omega$ en construisant par récurrence sur $\alpha$ une bijection de $\widetilde{T}_{\alpha}$ dans $\omega$ à l'aide d'une 
bijection de $\omega^{2}$ sur $\omega$. Quitte à choisir une bijection de $\omega^{2}$ sur $\omega$ nouvelle à chaque étape de la récurrence (ce qui revient à faire une permutation sur $\omega$ et qui est compatible avec $\preceq$ ), on peut assurer des conditions particulières sur $\mathcal{F}_{\alpha}$. Il n'est pas question de modifier $\widetilde{T}_{\alpha}$, donc il y a bien unicité de la base $\mathcal{F}_{\alpha}$ considérée.

Lemme 5.6. Soient $\mathcal{F}$ une base monotone sur $\omega, \alpha$ un ordinal dénombrable $\geq 1$ et \langle\rangle une bijection parfaite. Si pour tout $\beta<\alpha, A_{\beta}$ est la borne inférieure de $\mathcal{F}_{\beta}$, alors $A_{\alpha}=\langle\rangle \bigcup_{n \in A_{1}}\{n\} \times A_{[\alpha]_{n}}$ est la borne inférieure de $\mathcal{F}_{\alpha}$.

Le lemme précédant découle automatiquement de la définition d'une bijection parfaite.

Lemme 5.7. Soient $\widetilde{A} \in \mathcal{A}$ s et $\left(A_{n}\right)_{n \in \omega} \subset \mathcal{A}$ s. Alors, il existe une bijection parfaite \langle\rangle de $\omega^{2}$ sur $\omega$ telle que $A=\langle\rangle \bigcup_{n \in \widetilde{A}}\{n\} \times A_{n} \in \mathcal{A}$ s.

Démonstration. Pour définir une bijection parfaite \langle\rangle , il suffit de construire une suite $\left(\omega_{(n)}\right)_{n \in \omega}$ d'éléments de $2^{\omega}$ décroissante pour $\leq$ et formant une partition de $\omega$, puis de poser $\langle n, k\rangle=$ "le $k$-ième élément de $\omega_{(n)}$ " $\left((n, k) \in \omega^{2}\right)$.

Posons $f_{n}(i)=\inf \left\{\operatorname{card}\left(A_{n} \cap[0, j[) / / j ; j \geq i\}\right.\right.$ et $\widetilde{f}(i)=\inf \{\operatorname{card}(\widetilde{A} \cap$ $[0, j[) / / j ; j \geq i\}(i, n \in \omega)$; on a $\lim _{i \rightarrow \infty} f_{n}(i)=1$ et $\lim _{i \rightarrow \infty} \widetilde{f}(i)=1$, car $A_{n} \in \mathcal{A} s(n \in \omega)$ et $\widetilde{A} \in \mathcal{A} s$.

On définit, facilement, par récurrence sur $k$, une suite $\left(i_{k}\right)_{k \in \omega}$ telle que

$$
\begin{gathered}
i_{0}=0, \quad i_{k}-i_{k-1} \equiv 0(\bmod k), \\
\forall 0 \leq n \leq k, \quad f_{n}\left(\frac{i_{k}-i_{k-1}}{k}\right) \geq 1-\frac{1}{k} .
\end{gathered}
$$

Posons $\omega_{(n)}=\left\{i \in \omega ; \exists k \geq 1, i_{k-1} \leq i<i_{k}\right.$ et $\left.i-i_{k-1} \equiv n(\bmod k)\right\}$. Bien entendu, $\left(\omega_{(n)}\right)_{n \in \omega}$ est une suite décroissante pour $\leq$ sur $2^{\omega}$ et définit ainsi une bijection parfaite \langle\rangle de $\omega^{2}$ sur $\omega$.

Montrons que $A=\langle\rangle \bigcup_{n \in \widetilde{A}}\{n\} \times A_{n} \in \mathcal{A}$ s. Mais, pour tout $k, i \in \omega$, avec $i_{k}<i \leq i_{k+1}$,

$$
\begin{aligned}
\operatorname{card}(A \cap[0, i[) & \geq \operatorname{card}\left(A \cap \left[0, i_{k}[)+\widetilde{f}(k) \sum_{n=0}^{k} f_{n}\left(\frac{i_{k}-i_{k-1}}{k}\right) \frac{i-i_{k}}{k+1}\right.\right. \\
& \geq \operatorname{card}\left(A \cap \left[0, i_{k}[)+\widetilde{f}(k)\left(1-\frac{1}{k}\right)\left(i-i_{k}\right) .\right.\right.
\end{aligned}
$$

Comme $\widetilde{f}(k)$ est croissante, cette dernière inégalité est en fait vrai pour tout $i>i_{k}$, donc

$$
\mathrm{d}(A)=\lim _{i \rightarrow \infty} \operatorname{card}(A \cap[0, i[) / / i \geq \widetilde{f}(k)(1-1 / / k),
$$

et comme ceci est vérifié pour tout $k \in \omega, \mathrm{d}(A)=1$. 
Corollaire 5.8. Soit $\mathcal{F}$ une base monotone sur $\omega$. Si la borne inférieure de $\mathcal{F}$ appartient à $\mathcal{A} s$, alors pour tout ordinal dénombrable $\alpha \geq 1$, on a $\mathcal{F}_{\alpha} \preceq \mathcal{A} s$.

On déduit de ce corollaire la Proposition 5.4, car la borne inférieure $\omega \backslash\{0\}$ de $\mathcal{P}$ est dans $\mathcal{A} s$.

\section{Bibliographie}

[1] B. Aniszczyk, J. Burzyk and A. Kamiński, Borel and monotone hierarchies and extension of Rényi probability spaces, Colloq. Math. 51 (1987), 11-25.

[2] J. Arbault, Sur l'ensemble de convergence absolue d'une série trigonométrique, Bull. Soc. Math. France 80 (1952), 253-317.

[3] H. Becker, S. Kahane and A. Louveau, Natural complete $\Sigma_{2}^{1}$-sets in Harmonic Analysis, Trans. Amer. Math. Soc., to appear.

[4] G. Choquet, Sur les notions de filtre et de grille, C. R. Acad. Sci. Paris 224 (1947), $171-173$.

[5] S. Kahane, Ensembles de convergence absolue, ensembles de Dirichlet faibles et $\uparrow$-idéaux, ibid. 310 (1990), 335-337.

[6] —, - 个-idéaux de compacts et applications à l'analyse harmonique, Thèse, Univ. Paris 6, 1990.

[7] —, Antistable classes of thin sets in Harmonic Analysis, Illinois J. Math., to appear.

[8] A. S. Kechris, A. Louveau and W. H. Woodin, The structure of $\sigma$-ideals of compact sets, Trans. Amer. Math. Soc. 301 (1987), 263-288.

[9] K. Kuratowski, Topology I, Acad. Press, New York 1966.

[10] H. Lebesgue, Sur les fonctions représentables analytiquement, J. Math. Pures Appl. (6) 1 (1905), 139-216.

EQUIPE D'ANALYSE

UNIVERSITÉ PARIS 6

4, PLACE JUSSIEU

75252 PARIS CEDEX 05, FRANCE

Received 9 September 1991;

in revised form 10 March 1992 